\title{
Microfluidic integration of regeneratable electrochemical affinity-based biosensors for continual monitoring of organ-on-a-chip devices
}

\author{
Julio Aleman $\mathbb{1}^{1,4}$, Tugba Kilic ${ }^{2,4}$, Luis S. Mille $\mathbb{1}^{3}$, Su Ryon Shin ${ }^{3 凶}$ and Yu Shrike Zhang $\mathbb{\circledR}^{3 凶}$
}

\begin{abstract}
Organs-on-chips have emerged as viable platforms for drug screening and personalized medicine. While a wide variety of human organ-on-a-chip models have been developed, rarely have there been reports on the inclusion of sensors, which are critical in continually measuring the microenvironmental parameters and the dynamic responses of the microtissues to pharmaceutical compounds over extended periods of time. In addition, automation capacity is strongly desired for chronological monitoring. To overcome this major hurdle, in this protocol we detail the fabrication of electrochemical affinity-based biosensors and their integration with microfluidic chips to achieve in-line microelectrode functionalization, biomarker detection and sensor regeneration, allowing continual, in situ and noninvasive quantification of soluble biomarkers on organ-on-a-chip platforms. This platform is almost universal and can be applied to in-line detection of a majority of biomarkers, can be connected with existing organ-on-a-chip devices and can be multiplexed for simultaneous measurement of multiple biomarkers. Specifically, this protocol begins with fabrication of the electrochemically competent microelectrodes and the associated microfluidic devices $(\sim 3 \mathrm{~d})$. The integration of electrochemical biosensors with the chips and their further combination with the rest of the platform takes $\sim 3 \mathrm{~h}$. The functionalization and regeneration of the microelectrodes are subsequently described, which require $7 \mathrm{~h}$ in total. One cycle of sampling and detection of up to three biomarkers accounts for $\sim 1 \mathrm{~h}$.
\end{abstract}

Organ-on-a-chip systems have emerged as an enabling in vitro platform for drug screening by providing the biomimetic 3D microenvironments and dynamic cues that are typically lacking in conventional simplified cell cultures ${ }^{1}$. Individual organ models can be connected in the same manner in which they appear in the human body to further reproduce interactions among the multiple tissue types $^{2}$. Besides on-chip models of healthy tissues and organs, their diseased counterparts can also be generated to bridge the gap between animal models and humans, particularly for disease types that cannot be faithfully established in animals ${ }^{3}$. To date, almost all human tissues have been reproduced in organ-on-a-chip devices, ranging from cardiovascular ${ }^{4}$ and musculoskeletal ${ }^{5}$ systems to neuronal ${ }^{6}$, digestive $^{7}$ and renal systems ${ }^{8}$, as well as those in various disease states such as cardiomyopathy ${ }^{9}$, progeria $^{10}$ and cancer $^{11}$. These improved in vitro models of human physiology and pathophysiology have made it possible to screen pharmaceutical compounds at higher accuracy and to achieve precision medicine through personalizing chips using patient-derived materials ${ }^{12}$.

Despite great success of the organ-on-a-chip systems for broad applications in medicine, it is not always straightforward to characterize microtissue behaviors and their interactions with drug molecules. For example, the levels of biomarkers secreted by the microtissues are strongly indicative of their status, which change dynamically as the microtissues form, grow and mature, and as they experience external stimuli and undergo damage ${ }^{13-15}$. Often, the biomarkers are measured by off-chip methods such as enzyme-linked immunosorbent assay (ELISA), which necessitates the medium to be sampled at desired time points ${ }^{16-19}$. The repeated operations might disturb the microtissue responses within the microenvironments, and the typically large sampling volumes (comparing to the sizes of miniaturized microfluidic chip devices) inevitably limit the ability to continuously monitor the alterations in biomarker levels at elevated frequency ${ }^{20}$.

${ }^{1}$ Department of Bioengineering, Swanson School of Engineering, University of Pittsburgh, Pittsburgh, PA, USA. ${ }^{2}$ Center for Systems Biology and Department of Radiology, Massachusetts General Hospital Research Institute, Harvard Medical School, Boston, MA, USA. ${ }^{3}$ Division of Engineering in Medicine, Department of Medicine, Brigham and Women's Hospital, Harvard Medical School, Cambridge, MA, USA. ${ }^{4}$ These authors contributed equally: Julio Aleman, Tugba Kilic. ${ }^{凶}$-mail: sshin4@bwh.harvard.edu; yszhang@research.bwh.harvard.edu 
To this end, strategies to integrate biosensors with microfluidic chips that can be directly connected to organ-on-a-chip systems become critical to performing in situ, continual and low-volume biomarker analyses. In this protocol, we provide a detailed procedure describing our recently developed approach for the fabrication of electrochemical (EC) affinity-based biosensors and their integration with microfluidic chips ${ }^{13,21,22}$.

\section{Development of the approach}

To achieve our strategy, EC affinity-based biosensors are a good candidate because of their unique advantages such as excellent limit of detection, wide linear detection range, label-free detection ability, scalability to monitor multiple biomarkers and relatively simple operations and fabrication processes compared with other existing biosensor systems ${ }^{14,21-23}$. Importantly, electrodes-a major part of ECbased biosensor systems-are simple to miniaturize through microfabrication processes; hence, microfabricated electrodes facilitate integration into organ-on-a-chip systems via microfluidic platforms (Fig. 1). Also, EC biosensors can be easily expanded with an array of microelectrodes that allow for the monitoring of multiple biomarkers using small sample volumes, resulting in accurate and efficient monitoring of biological behaviors and responses of microtissues in organ-on-a-chip systems ${ }^{13}$.

In an ideal situation, the integrated affinity-based biosensors in an organ-on-a-chip system should continually monitor biomarkers to evaluate the dynamic behaviors of the microtissue for an extended period of time. To achieve this strategy, one of the major drawbacks of existing affinity-based biosensors $^{24,25}$ should be solved: that is, a lack of multipoint detection ability using single affinitybased biosensors due to surface saturation of the sensor when bioreceptors (antibodies or aptamers) repeatedly capture target biomarkers via their strong binding interaction. Therefore, various surfacecleaning processes have been developed for the regeneration of saturated microelectrodes that can allow multipoint detection strategies to function in a continual manner without replacing saturated biosensors. However, most cleaning methods require harsh cleaning solutions (low- or high-pH solutions) and time-consuming procedures that can damage the biosensor surface. In particular, damage to bioreceptors (antibody or aptamer) during the regeneration processes can affect their ability to capture target biomarkers, resulting in decreased sensitivity and selectivity. To avoid biosensor surface damage in the regeneration process, robust biosensors are required when harsh cleaning processes are applied. In this protocol, we describe the fabrication of robust EC affinitybased biosensors along with an improved EC cleaning method that will maintain EC oxidation signals on bare microelectrodes for up to 25 regenerations. This two-step regeneration strategy can also remove all molecules from the microelectrode surface, allowing for subsequent reimmobilization of fresh bioreceptors, with reproducible sensitivity/selectivity of the same biosensor for up to five regeneration cycles (Figs. 1 and 2). The reusable EC affinity-based biosensors can be readily integrated into organ-on-a-chip platforms through microfluidic chips to enable continual and long-term measurements of secreted biomarkers from microtissues upon drug, chemical or other exposures.

Microfluidic integration of the EC affinity-based biosensors is key to their successful application in organ-on-a-chip platforms ${ }^{20}$. This protocol further discusses the design of microfluidic chips that allow for seamless integration with microfabricated electrodes. Enabled by built-in microfluidic channels with digitally controlled pneumatic valves, automated liquid routing can be achieved to facilitate a series of events including microelectrode functionalization, medium sampling and microelectrode regeneration, all completed on-chip with minimum manual operations (Fig. 3). The microfluidic chip design enables direct integration of these EC affinity-based chips with most existing organ-on-a-chip platforms through simple coupler connections or a microfluidic breadboard (see the 'Applications' section below), making them almost universally adaptable to biomarker measurements

Fig. 1 Schematic representation of the electrode fabrication and functionalization. a, Diagram of the two-stage fabrication steps of the microelectrode. With the use of two shadow masks (CE/WE and RE), an initial $20 \mathrm{~nm}$ layer of Ti is deposited, followed by a similar layer of Pd. For the CE/ WE mask, a $500 \mathrm{~nm}$ layer of Au is deposited, and a similarly thick layer of Ag for the RE mask. b, Au WE, Au CE and Ag RE-microfabricated electrode functionalization and regeneration steps for antibody-based and aptamer-based biosensing of soluble antigens. c-i, Nyquist plots showing EIS data collected from the bare or modified microfabricated electrodes following corresponding modifications. Each Nyquist plot represents the ElS response for aptamer-based (c) and antibody-based (d) functionalization steps, regeneration steps (e) and calibration measurements obtained from 1-100 ng $\mathrm{mL}^{-1}$ of GST- $\alpha(\mathbf{f})$ and $0.01-50 \mathrm{ng} \mathrm{mL} \mathrm{m}^{-1}$ of CK-MB (h); Calibration curves drawn with respect to normalized $R_{\mathrm{ct}}$ data from three independent electrode measurements for GST- $\alpha(\mathbf{g})$ and CK-MB (i), $n=3$; data expressed with all data points and as mean \pm standard deviation. All electrochemical measurements are done in $50 \mathrm{mM} \mathrm{K}_{3} \mathrm{Fe}(\mathrm{CN})_{6}$ at RT. $\mathbf{j}-\mathbf{I}$, AFM images of the Au electrodes at $1 \mu \mathrm{m}^{2}$ scan range before (j) and after $(\mathbf{k})$ antibody and aptamer (I) functionalization. 

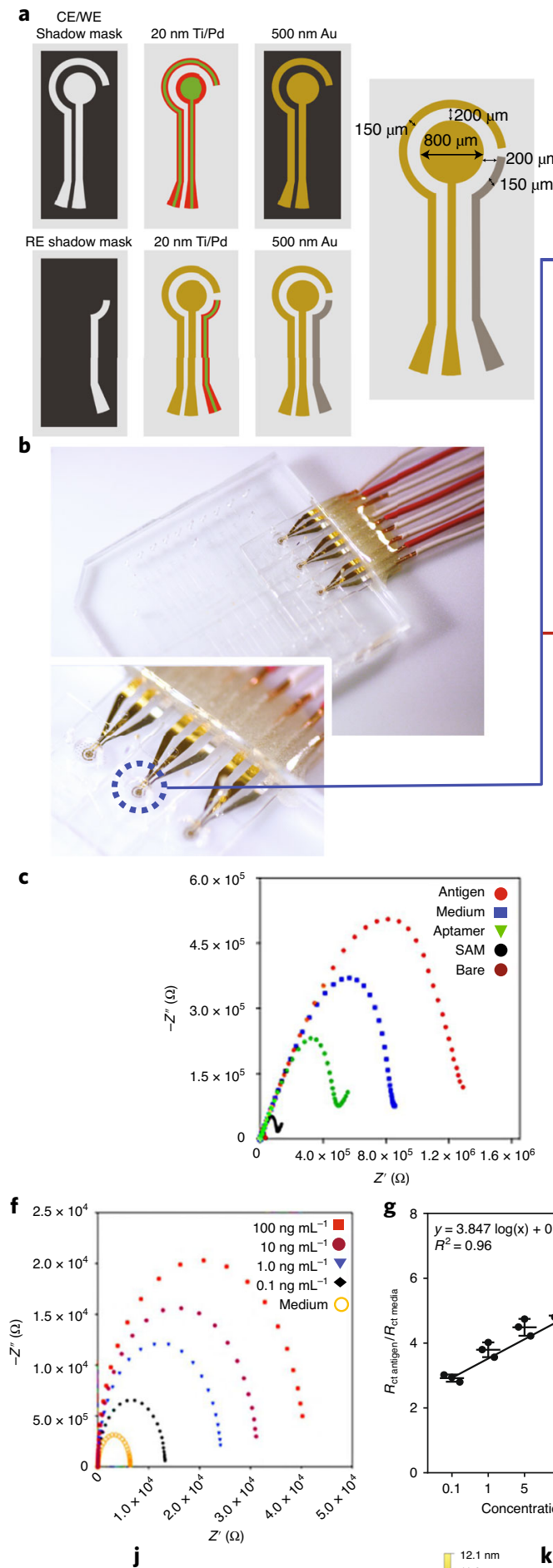

j

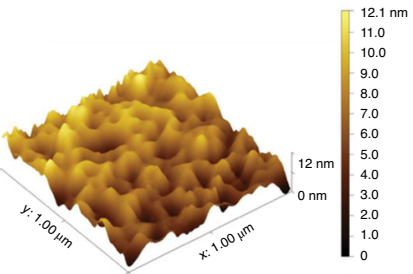

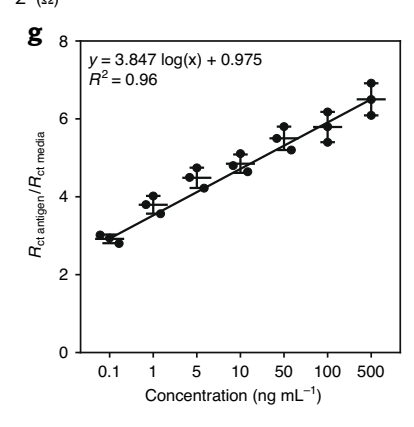
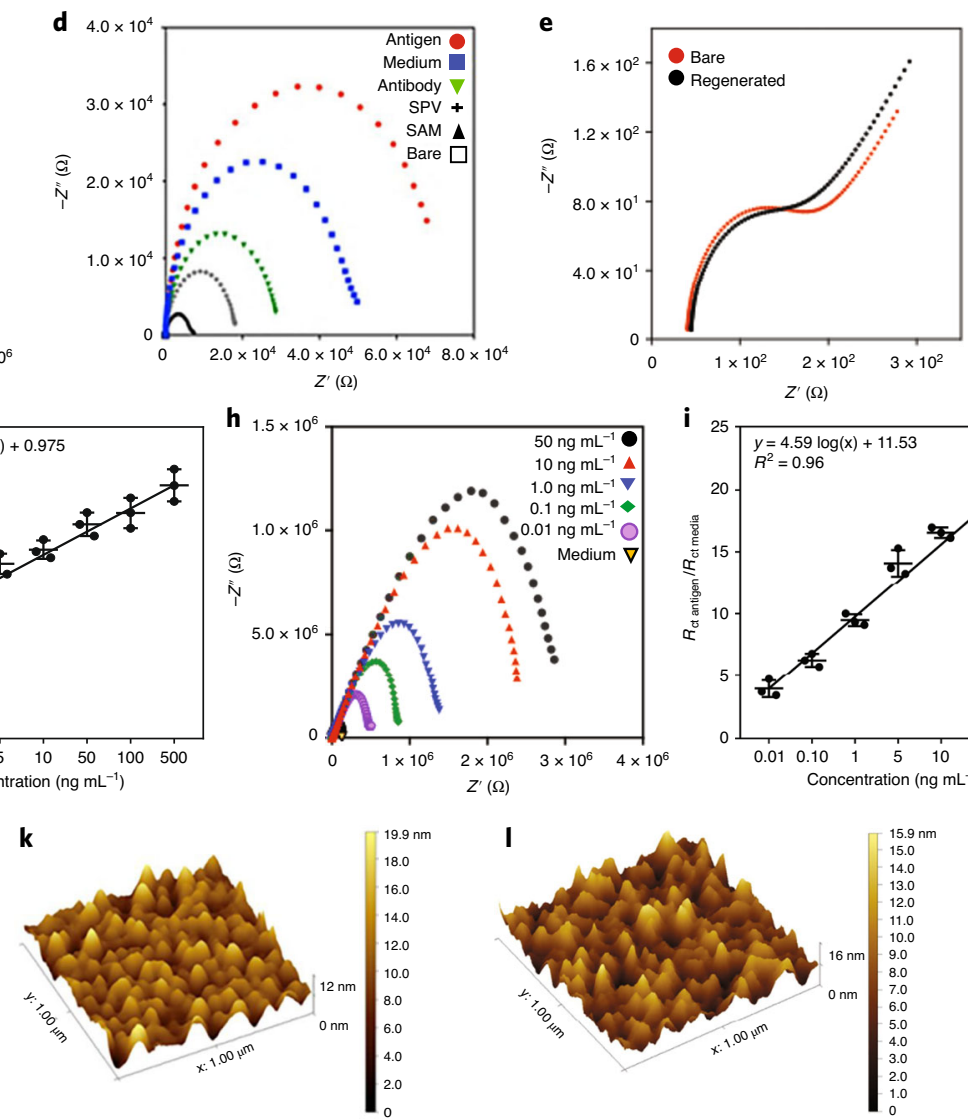
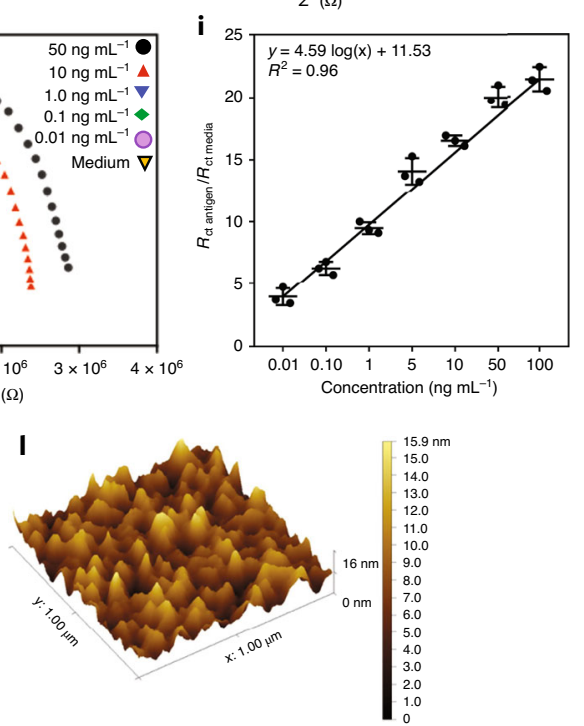


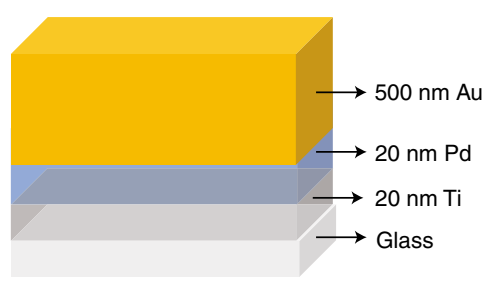

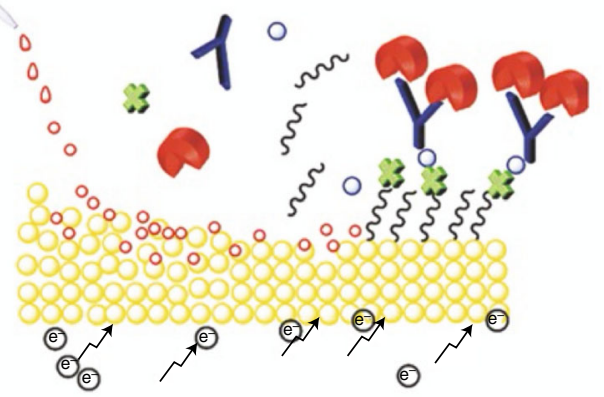

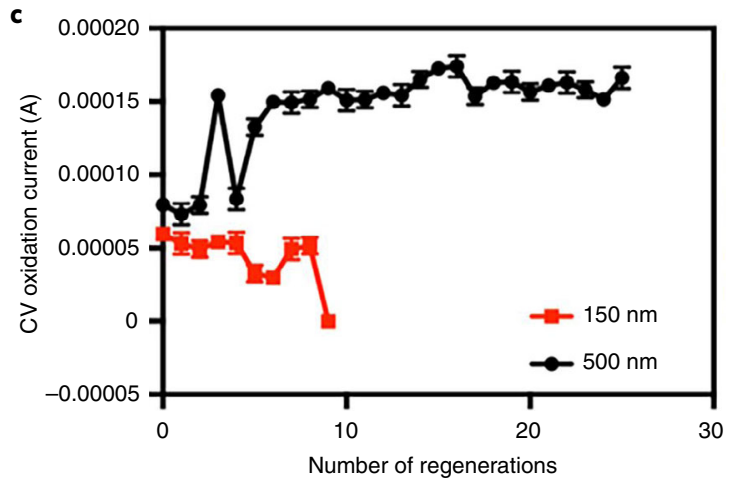

d

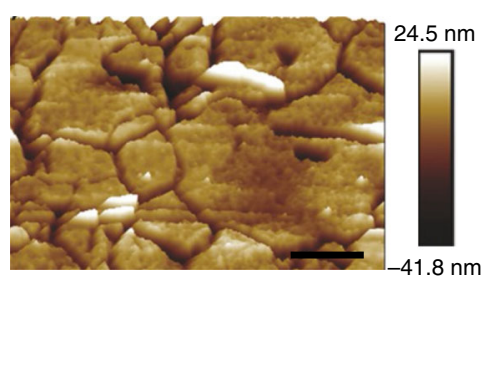

C

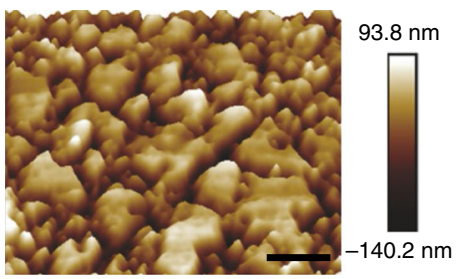

f

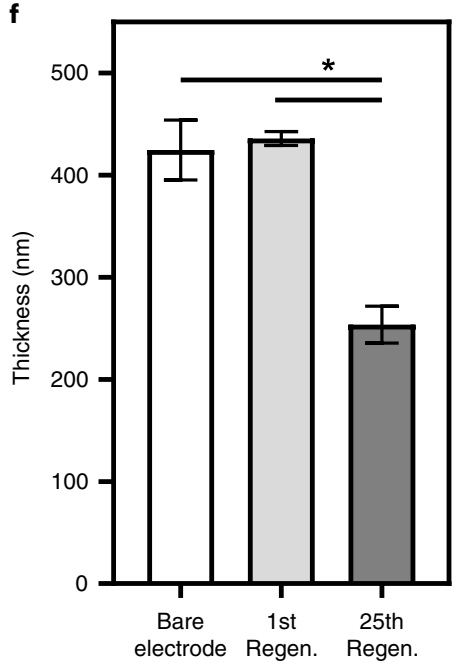

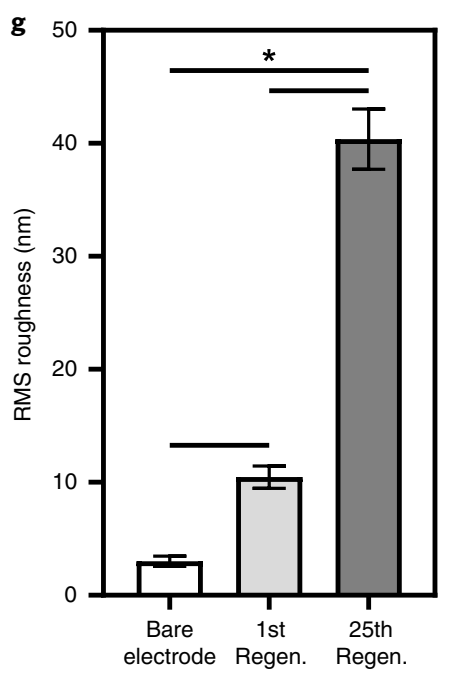

h

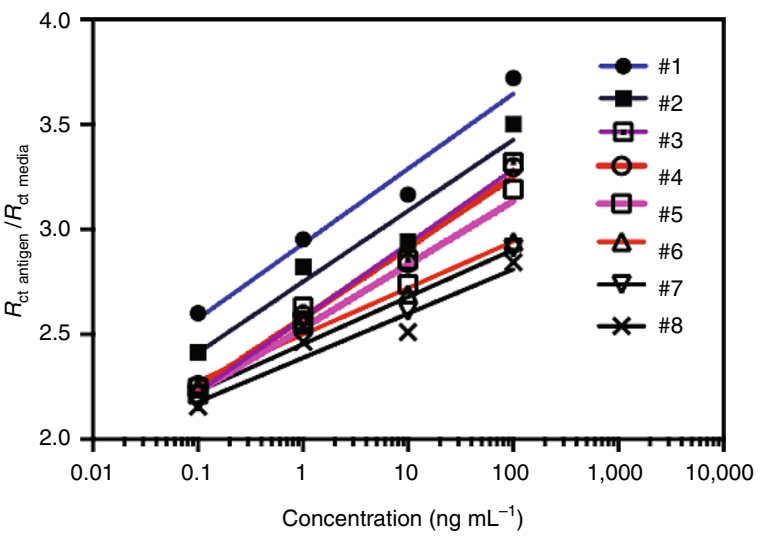

Fig. 2 | Effect of Au electrode thickness on reproducibility, sensitivity and selectivity upon multiple regeneration cycles. a,b, Schematic representation of Au microelectrodes with different Au layer thicknesses (a) and flow-assisted dual-step electrode regeneration (b). b. Potential sweep at the Au electrode and flow of washing solution remove the immobilized molecules. SAM (black line), SPV (green cross sign), primary antibody (blue) and target molecules (red). c, Changes in oxidation current values with respect to the number of regeneration cycles at the two different Au microelectrode thicknesses, $n=3$; data expressed as mean \pm standard deviation. d,e, AFM images of Au microelectrode surface after 1st (d) and 25 th (e) regeneration. Scale bars in $\mathbf{d}$ and $\mathbf{e}, 1 \mu \mathrm{m}$ and $\mu \mathrm{m}$, respectively. $\mathbf{f}, \mathbf{g}$, Histograms demonstrating changes in the Au microelectrode thickness (f) and roughness ( $\mathbf{g}$ ) upon 25 regeneration cycles, $n=7$; data expressed as mean \pm standard deviation, ${ }^{\star} P<0.0001$. $\mathbf{h}$, Au microelectrode calibration curves at different regeneration cycles. The thickness of the microelectrodes used in $\mathbf{d}-\mathbf{h}$ was $500 \mathrm{~nm}$. Figure adapted with permission from ref. ${ }^{21}$.

from engineered microtissue models and organoids. EC affinity-based biosensing chips can also be designed to suit different purposes, such as single-electrode format for quantification of one biomarker (Fig. 3d), single-use (until saturation occurs) format without regeneration (Fig. 3e) and multielectrode format for multiplexed biomarker detections with regeneration capability (Fig. 3f). In this protocol we will only discuss the single-microelectrode format (Fig. 3d), as the other two systems can be readily derived from this format by reducing or increasing the number of microchannels built into the microfluidic chips.

Besides the microfluidic chip that integrates the microelectrode for automated electrode functionalization, biomarker quantification and microelectrode regeneration, this protocol also discusses 


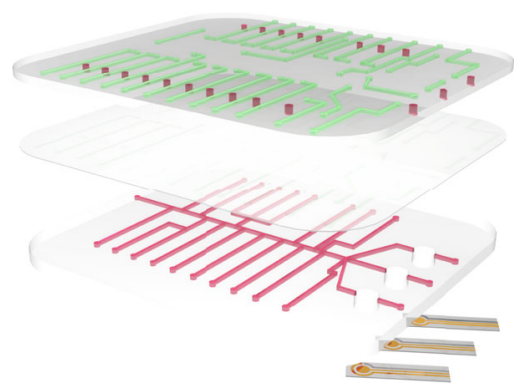

d

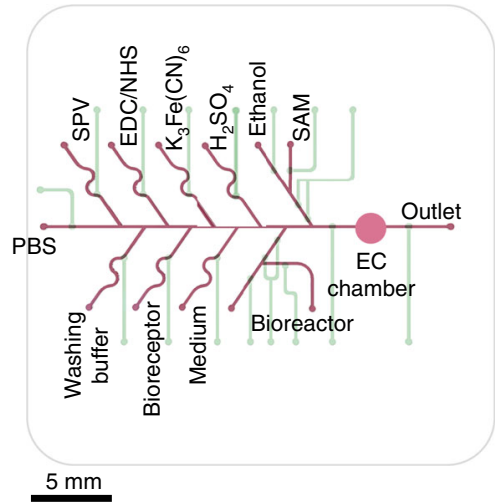

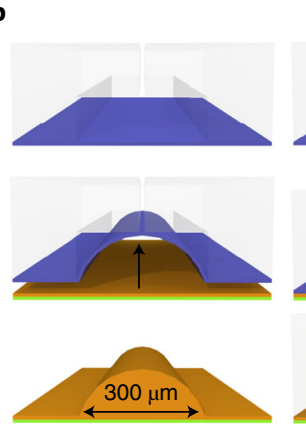

e
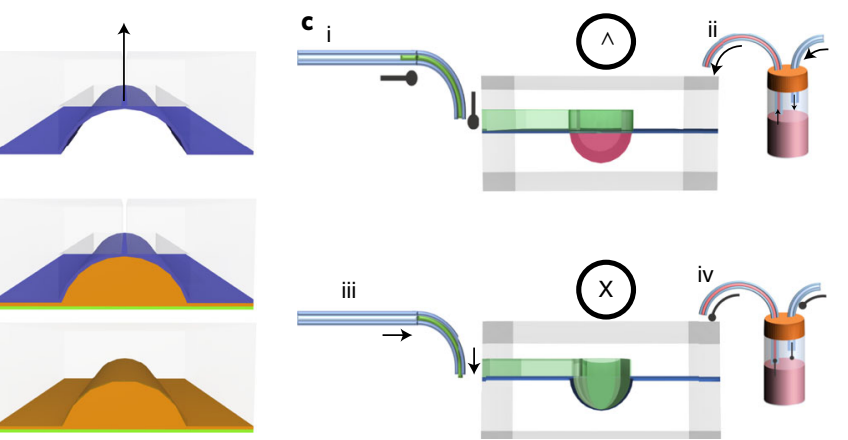

'

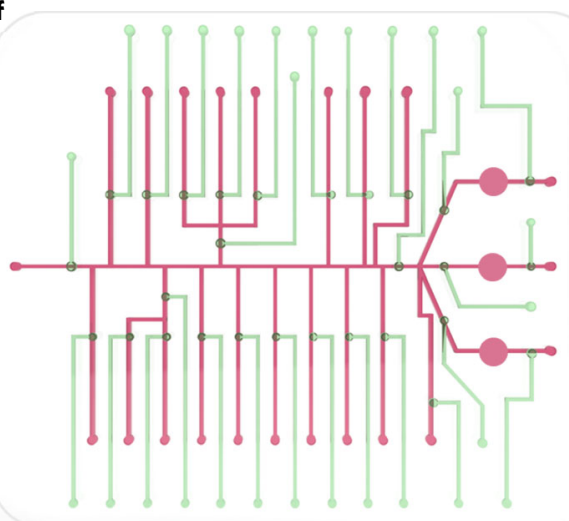

Fig. 3 | Assembly and valve configuration of microfluidic EC affinity-based biosensor chips (see Supplementary Data 1 for designs). a, Diagram showing the layers that compose the EC affinity-based biosensor chip: valve (green), membrane (middle, transparent), flow (red) and microelectrode (bottom right) layer. b, Fabrication of hemicylindrical flow layer channels. This specific configuration allows for a more reliable valve functionality. A squared PDMS replica of the flow layer is plasma-bounded to a 20 - $\mu$ m-thick PDMS membrane (blue). Negative pressure with a vacuum pump (upward arrow) is applied through the inlet ports, causing the membrane to deform into rectangular features. A SU8 (orange) replica of the curved channels is cured by UV exposure. PDMS replica of the hemicylindrical flow layer can now be further created. c, The valve system works through pneumatic pressure, where (i) if a solenoid valve is closed (oval arrow), the pressure on the membrane (blue) is released (ii) and the flow channel solenoid is opened (stealth arrow) to restart the pneumatic pressure in the reservoir (orange-capped vial) and encourage liquid flow (circumflex). (iii) When the solenoid valve is open (stealth arrow), the liquid on the valve channel (green) deforms the membrane into the fluid channel, blocking the passing of solutions, and (iv) the stream of the solution is further paused (circled X) by turning off (oval arrow) the corresponding solenoid that drives the pneumatic pressure inside the reservoir. The custom MATLAB code can coordinate the valve/flow solenoid dynamics to allow the orchestrated operation of the functionalization, sampling, detection and regeneration procedures. d-f, Illustration of three representative EC affinity-based biosensors chips: EC chip possessing automated regeneration capability with a single-electrode configuration (d), EC chip without regeneration configuration (e) and EC chip possessing automated regeneration capability with a multielectrode configuration (f).

the design of a microfluidic breadboard system for convenient interfacing of the EC affinity-based biosensing chip with one or multiple microtissue models. The breadboard is a central liquid-routing system containing user-defined microfluidic channel arrangements and pneumatic valves, with inlets/ outlets built into different segments to connect to side modules (Fig. 4). These side modules can include the EC affinity-based biosensing chips, bioreactors hosting engineered microtissue models or organoids, bubble traps and medium reservoirs. As such, the automated liquid routing taking place in the central breadboard will lead to well-defined flow sequence in the side modules and controlled perfusion of, for example, the EC affinity-based biosensing chip for medium-sampling at desired time points and intervals. The breadboard configuration enables flexible integration of the EC affinitybased biosensing chip with most of the standard and existing microfluidic units used in organ-on-achip platforms.

\section{Overview of the procedure}

In this protocol, we describe in detail the preparation of the microfabricated electrodes (Steps 1-10); microfluidic chip design and fabrication (Steps 11-64); the integration of the microelectrode with the microfluidic chip (Steps 65-95); integration with the breadboard and bioreactor (Steps 109-136); procedures for on-chip microelectrode functionalization (Steps 137-166); procedures for on-chip medium sampling and biomarker measurement (Steps 167-174); and finally procedures for on-chip 
a
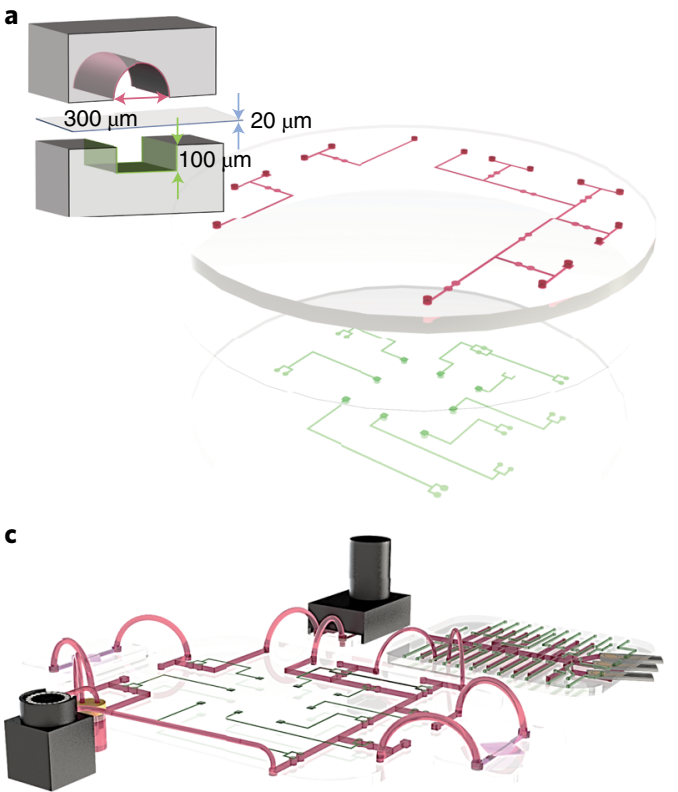

b

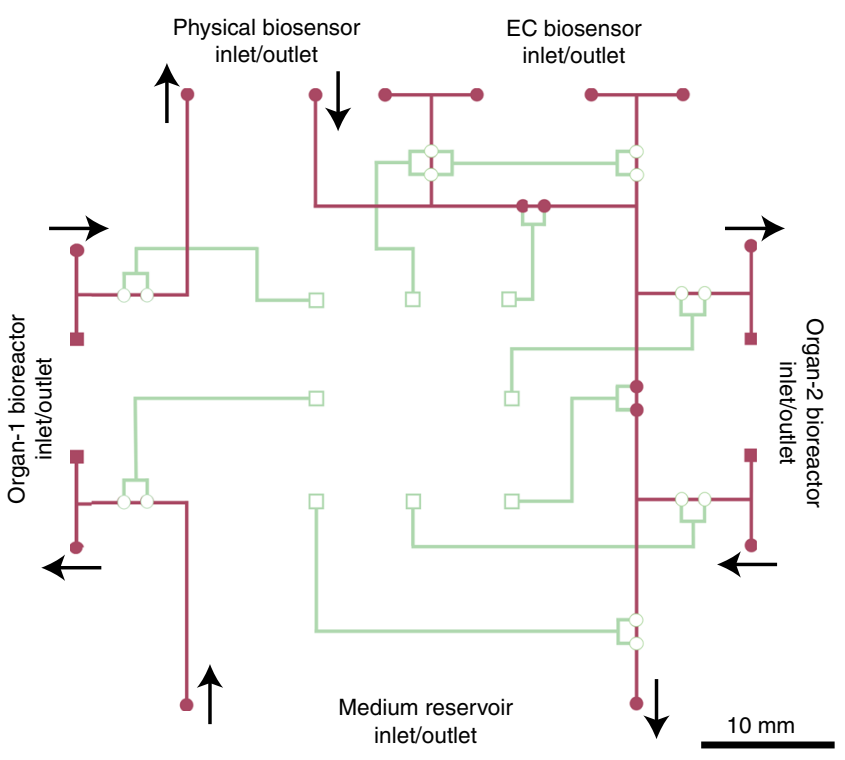

d

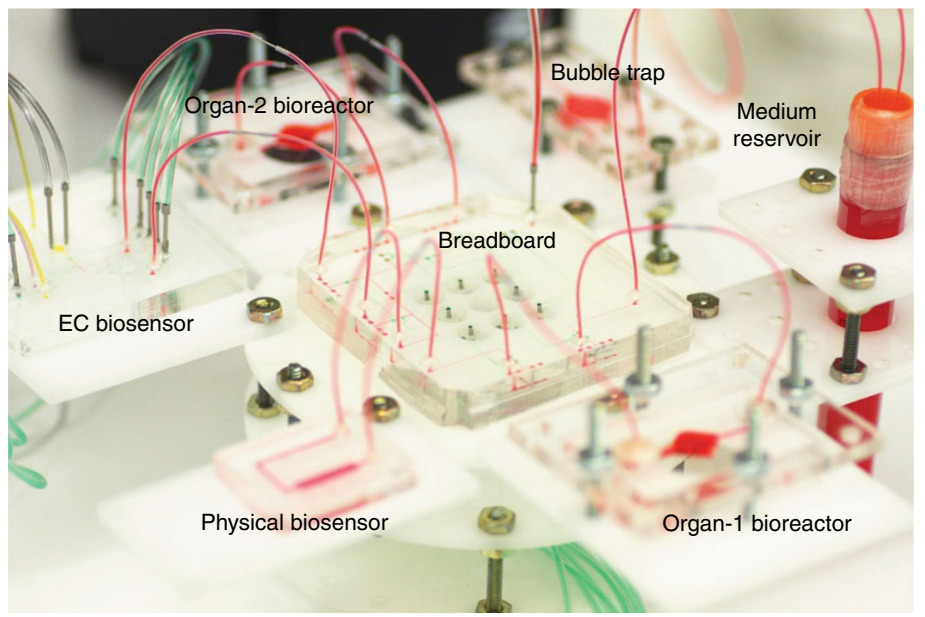

Fig. 4 | Diagrams of the assembly of the breadboard and its connections with side modules (see Supplementary Data 1 for designs). a, The breadboard consists of three layers: the valve, membrane and fluidic layer. All layers are plasma-bounded together. b,c, The closed-loop configuration of the breadboard can interconnect two organ bioreactors, with a physical sensor and the EC affinity-based biosensor chip in between organs, which can be further custom-configured as needed. d, Photograph showing a typical multisensor-integrated multi-organ-on-chips platform. The red color shows medium circulation, and the green color indicates the valve connections. Figure adapted with permission from ref. ${ }^{13}$.

microelectrode regeneration (Steps 175-180). We initially reported on an automatized antibody/ aptamer-based single EC biosensor to detect cardiac biomarkers, with nonregeneration capabilities ${ }^{22}$. Following this work we developed another EC biosensor with regeneration capabilities for continuous detection of liver-associated secretomes via antibody affinit $y^{21}$. Finally, we reported on a multiplexed EC biosensor capable of continuous detection of hepatic and cardiac biomarkers from microbioreactor modules integrated into a microfluidic breadboard controller ${ }^{13}$. These publications summarize the conditions and detailed fabrication of our liver and cardiac tissues, as well as the implementation of the supplementary physical biosensors. This protocol presents the necessary steps for the fabrication, assembly and functionalization of an EC affinity-based biosensing chip, along with the necessary steps for automatization operations through the breadboard and other components. We recommend that users utilize a bench-top or customized incubator, whichever allows the peripheral electronical components and pneumatic tubing to be easily accessible. 


\title{
Applications
}

The affinity-based microfluidic regeneratable EC biosensors described in this protocol can be used for the detection of cell secretomes from any cell culture medium, providing that corresponding bioreceptors are available. Monitoring protein biomarker levels with these biosensors can be useful for drug toxicity and drug screening studies as well as monitoring changes in cell culture microenvironments upon most types of external stimuli such as temperature, $\mathrm{pH}$ and osmolarity, among others, in both planar and 3D culture systems.

For example, isolation of single organs from a multiorgan tandem series has been established as a toxicology validation technique ${ }^{16,26}$, as well as fixed parallel series of multitissue setups for cell migration studies ${ }^{27,28}$. The automated platform allows for multibioreactor connectivity, with reversible bypass capabilities and programable flow parameters. In addition to flow control, the modular competence of the breadboard to intercalate microbioreactors and biosensor chips, plus the compliance of a wide range of molecules to the affinity-based biosensing platform, may allow for direct measurements of secretomes from one microbioreactor before the next one without systematic disruptions. These capacities boost monitoring of cell cultures, including organ-on-a-chip systems and data collection, to levels unattainable before.

The same method can also be extended to beyond cell culture monitoring and essentially any measurements based on affinity, such as detection of viral materials, including those of severe acute respiratory syndrome coronavirus 2 (SARS-CoV-2), in bodily fluids and environmental wastewater.

\begin{abstract}
Alternative methods
Detection of protein biomarkers can also be achieved via ELISA or various other biosensing platforms with different transduction mechanisms, such as optical, EC and piezoelectric ${ }^{29}$. Depending on the application, monitoring can either be on-line or endpoint. On-line monitoring requires continual or repeated operations over extended periods of time, which is a capacity lacking in most of the manual methods including ELISA as well as in most other automated biosensing strategies, making them more suitable for endpoint or short-term assays. Optical biosensors may be alternative tools for online monitoring. However, integration of biosensing platforms to organ-on-a-chip systems is another challenge since there are important constraints to take into account such as the minimum sampling volume and hardware/software automation. In this regard, the EC method provides advantages over other transduction mechanisms, because the microelectrodes are easily miniaturized while the design of portable EC workstations provides flexible solutions for integration purposes.
\end{abstract}

\section{Limitations of the protocol}

While the EC affinity-based biosensing chips can be almost universally interfaced with any existing organ-on-a-chip platforms, there are several limitations associated with the technology. In the current design, the regeneration can take up to $4 \mathrm{~h}$, making it difficult to continuously perform biomarker measurements within this time frame using a single chip. Nevertheless, this limitation can be addressed by modifying the breadboard to integrate multiple EC affinity-based biosensing chips that can operate in tandem, that is, when one is under regeneration, the others are still functioning, ensuring continual biomarker monitoring at higher frequencies. Another limitation lies in the fact that the current microfluidic chips are still reliant on polydimethylsiloxane (PDMS), which might exert negative effects on biomarker adsorption and/or absorption. This challenge can be addressed by switching to whole-thermoplastic chips. We have recently reported on the development of wholethermoplastic microfluidic devices containing various key microfluidic functional units relying also on pneumatic valves, such as normally open valves, normally closed valves, micro-check valves and micropumps $s^{30,31}$. Additional recent efforts have further revealed the possibility of using 3D printing to produce Quake-style microvalves within thermoplastic-like materials ${ }^{32}$. The regeneration requires full removal of a layer of the material from the surface of a microelectrode, limiting the lifetime of the device and the number of detections up to 25 regeneration cycles for bare microelectrodes, or $\sim 5$ regeneration cycles for the functionalized biosensors. One solution is to start with a thicker microelectrode. In the case of a sudden, substantially elevated level of the target biomarker, the platform can immediately saturate, and the value above the detection range can be missed. In addition, as an affinity-based biosensing platform, we are limited by the number of available antibodies and their corresponding epitopes. Aptamers have become a great alternative ${ }^{22}$, and we have also described a method to implement them in our system. 


\section{Experimental design}

Fabrication of microelectrodes

We typically use a three-electrode-configuration microelectrode with reference electrode (RE), counter electrode (CE) and working electrode (WE) as the basis of the affinity-based biosensor (Fig. 1a). The fabrication is done in two steps by electron-beam evaporation through $0.25 \mathrm{~mm}$-thick shadow masks (see Supplementary Data 1 for the designs) for creating Au (WE, CE) and Ag (RE) patterns on a $25 \times 25 \mathrm{~mm}^{2}$ glass substrate precleaned by oxygen plasma. In the first step, $800 \mu \mathrm{m}$ diameter WE and $150 \mu \mathrm{m}$-wide $\mathrm{CE}$ are patterned in $500 \mathrm{~nm}$-thick Au over a $20 \mathrm{~nm}$-thick Pd, followed by a $20 \mathrm{~nm}$-thick Ti. The second shadow mask enables the deposition of a $500 \mathrm{~nm}$-thick Ag RE patterning over a similar $10 \mathrm{~nm}$-thick Ti/Pd on the same glass substrate. The distance between $\mathrm{WE}$ and $\mathrm{CE} / \mathrm{RE}$ is $200 \mu \mathrm{m}$. The microfabricated electrodes are enclosed in a furnace at $300{ }^{\circ} \mathrm{C}$ for $6 \mathrm{~h}$ to achieve annealing. Scanning electron microscopy (SEM) and atomic force microscopy (AFM) are used for surface characterizations of the microfabricated electrodes (Fig. 1j-1).

\section{Principle of microelectrode functionalization and EC detection}

We provide a versatile functionalization strategy that incorporates biorecognition elements for capturing protein biomarkers secreted to the cell culture medium (Fig. 1c,d). Microfabricated Au WE surface is first coated with 11-mercaptoundecanoic acid (11-MUA) and then incubated with $N$-ethyl$N^{\prime}$-(3-dimethylaminopropyl) carbodiimide (EDC)/ $N$-hydroxysuccinimide (NHS) crosslinker solution to convert the carboxylic end of 11-MUA to an NHS ester for coupling primary amines in streptavidin (SPV)- or amine-terminated aptamers. Upon creating uniform patterns of SPV molecules on the WE surface, the biotinylated capture probe is immobilized via strong biotin-SPV interaction that enables a flow-resistant bioreceptor surface. The selectivity of the biosensor surface is assured by a blocking step where nonspecific binding of nontarget proteins is eliminated through incubation of fresh cell culture medium that is used for the target microtissue culture and is rich in proteins such as bovine serum albumin (BSA) or other components.

Detection and quantification of protein biomarkers interacting with the biotinylated probe relies on a label-free EC method that enables monitoring the change of interfacial electron-transfer kinetics of the redox probe $\left[\mathrm{Fe}(\mathrm{CN})_{6}\right]^{4-/ 3-}$ upon biorecognition reaction. EC impedance spectroscopy (EIS) is employed to measure the impedance value after each sample incubation. The Randles equivalent circuit model is used to fit the raw EIS data collected over a wide frequency range and is comprised of electrolyte or solution resistance, $R_{\mathrm{s}}$, between WE and RE, the double layer capacitance, $C_{\mathrm{dl}}$, and the Faradaic impedance at the electrode-electrolyte interface that is subdivided into the charge transfer resistance, $R_{\mathrm{ct}}$, and the Warburg impedance, $W$. The resulting data are presented by Nyquist plots in which real and imaginary parts of impedance are plotted to clearly differentiate charge transfercontrolled and diffusion-controlled processes that are related to semicircular and linear parts of the plots, respectively. Increased capture of antigens on the WE surface upon successive sample incubation results in gradual increase in $R_{\mathrm{ct}}$ values due to shielding of the redox probe by antigens. The increase in $R_{\mathrm{ct}}$ values continues until the point where the WE surface is saturated to the capture of more antigens and needs to be regenerated for further measurements. In addition to impedance measurements, AFM images taken at bare (Fig. 1j), as well as antibody- (Fig. 1k) and aptamer(Fig. 11) functionalized, microelectrodes also indicate the successful functionalization of the bioreceptors. According to AFM images, the mean surface roughness of the bare microelectrode $(1.79 \mathrm{~nm})$ is increased after antibody $(2.75 \mathrm{~nm})$ and aptamer $(2.53 \mathrm{~nm})$ functionalization.

\section{Incorporation of different types of capture probes on EC affinity-based biosensors}

Our versatile functionalization strategy enables incorporation of bioreceptor molecules in the form of proteins and nucleic acids such as antibodies and aptamers, respectively ${ }^{21,22}$. Depending on the selected biomarker target, detection medium or duration of monitoring, the two capturing probes can be selected accordingly (Fig. If-h). Aptamers are widely employed when it comes to the matter of high selectivity and stability over time, as well as change in chemical composition, since they are meticulously designed by the systematic evolution of ligands by exponential enrichment (SELEX) method $^{33}$ so that they can bind to their targets with very high affinities and remarkable dissociation constants ranging from picomolar to nanomolar. Most of the aptamer-based biosensing platforms employ methylene blue-functionalized aptamers as molecular switches that go through a conformational change upon target binding and induce changes in EC signals. These molecular switchbased platforms hold great potential for monitoring in organ-on-a-chip systems because of their 
simplicity at the surface functionalization $\operatorname{step}^{34,35}$. They are, however, not covered in our protocol because of their incompatibility with the generalized procedures described. On the other hand, aptamer synthesis is a tedious, time-consuming process requiring more resources and is sometimes hindered by the limited nucleobase repertoire.

\section{Method for regeneration of EC affinity-based biosensors}

Long-term monitoring of protein biomarkers in cell culture medium is achieved via successful regeneration of the biosensor surface upon saturation after several incubations. A two-step EC regeneration process is employed to remove all the molecules immobilized to the biosensor surface during functionalization and biomarker detection (Fig. 1b,e). The biosensor surface is first flushed with $10 \mathrm{mM} \mathrm{H}_{2} \mathrm{SO}_{4}$ between electrical sweep potentials of $0.0 \mathrm{~V}$ and $+1.8 \mathrm{~V}$ at a scan rate of $100 \mathrm{mV} \mathrm{s}^{-1}$, a sensitivity of $10^{-2} \mathrm{~A} \mathrm{~V}^{-1}$ and a $0.001 \mathrm{~V}$ sampling interval, then followed by a $50 \mathrm{mM} \mathrm{K} \mathrm{K}_{3} \mathrm{Fe}(\mathrm{CN})_{6}$ flow with another potential sweep between $+1.0 \mathrm{~V}$ and $0.0 \mathrm{~V}$ at a scan rate of $200 \mathrm{mV} \mathrm{s}^{-1}$, a sensitivity of $10^{-4} \mathrm{~A} \mathrm{~V}^{-1}$ and a sampling interval of $0.001 \mathrm{~V}$. Continuous flows of the respective solutions over the courses of potential sweeps prevent readsorption of the detached molecules to the sensor surface and increase the efficiency of regeneration. Compared to nonflow-regeneration conditions, where the solution flow is stopped during potential sweep, the continuous flow regeneration is proven to create a smoother and more uniform surface that leads to a higher number of regenerations with a higher sensitivity for a longer monitoring period ${ }^{21}$. Another factor that is shown to substantially affect the performance of the regeneration is the thickness of the Au layer deposited on the Pd layer (Fig. 2a,b). Conductivity values of the regenerated and as-fabricated electrodes suggest higher discrepancy for the $150 \mathrm{~nm} \mathrm{Au}$ layer with respect to the $500 \mathrm{~nm}$ Au layer (Fig. 2c), since the thickness (Fig. 2d-f) and the roughness (Fig. 2d,e,g) of the electrodes change through the regeneration cycles when the two-step regeneration protocol is applied. The bare Au electrode with $150 \mathrm{~nm}$ thickness could not measure the signals after the $\sim 10$ th regeneration, while the bare Au electrode with $500 \mathrm{~nm}$ thickness could perform up to the 25th cycle of regeneration without substantial loss in the conductivity (Fig. 2c). Sensitivity of the functionalized, affinity-based biosensors seemed stable with $\sim 5$ cycles of repeated regeneration (Fig. 2h).

\section{Strategies for microfluidic integration and automation}

We provide the design of a microfluidic chip device that contains a microchamber to interface the microelectrode and a set of microchannels with built-in pneumatic valves to achieve digitally controlled liquid routing (Fig. 3a). This microfluidic chip features a three-layer configuration permanently bonded following plasma treatment. While the microfluidic channels in both the top pressure layer and the bottom fluid layer are fabricated using the standard soft lithography approach, the square microfluidic channels in the bottom layer of the chip are further converted to a convex hemicylindrical shape to facilitate complete pneumatic sealing (Fig. $3 \mathrm{~b})^{36}$. Between the two layers, a thin PDMS membrane $\left(20 \mu \mathrm{m}\right.$ in thickness) is sandwiched, functioning as the pneumatic valve $\mathrm{e}^{37}$. During an operational procedure, a pneumatic pressure is applied from the desired microchannel(s) in the valve layer of the device, which then pressurizes the thin membrane at the intersection to deform, blocking the microfluidic channel layer of the chip (Fig. 3c). The pneumatic microchannels are controlled by compressed nitrogen gas connected through polyvinyl chloride (PVC) tubing actuated via a digitized valve-controlling system (https://sites.google.com/site/rafaelsmicrofluidicspa ge/). The valve-controlling system is operated in full automation using a custom-defined MATLAB program (available via https://shrikezhang.com/publications/opensource; Electrochemical Biosensing, or in ref. ${ }^{13}$ ) written to specify the intervals of application and release of the pneumatic pressures by opening/closure of the Festo valve array. Similarly, the fluid movement in the bottom layer of the chip is also controlled automatically using pneumatic pressures. Each inlet of the microfluidic channels is connected to a Teflon tube linked to the outlet of a sealed reagent reservoir (Fig. $3 \mathrm{c}$ ), where the inlet of the reservoir when pressurized with compressed nitrogen gas drives the reagent to infuse into the fluidic channel of the chip. Coupled operations of pressure release on the reservoir and pressure application on the valve lead to flow cessation in the chosen microfluidic channel; on the contrary, sequential release of the pressure on the valve and application of the pressure on the reservoir restore the reagent flow. In our experiments, the valve control to seal the microchannels usually requires a high pneumatic pressure in the range of 10-20 PSI, while a lower range of pressures (1-5 PSI) is used to drive the reagent flows with desired flow rates. 
Breadboard design and modular integration

The breadboard has a similar configuration with the microfluidic chip for microelectrode integration. It comprises three layers that allow for automation control of the valves and the fluid flows (Fig. 4a). The primary difference lies in the arrangement of the microchannels, which should be customized to accommodate the need for the peripheral modules. In the example (Fig. $4 \mathrm{~b}-\mathrm{d}$ ), the breadboard features on-board microchannels that link to two bioreactors hosting two different engineered microtissues/organoids, a microfluidic EC affinity-based biosensing chip, a physical/chemical sensing chip and a medium reservoir. This configuration can be conveniently customized depending on the specific side modules to be included, where the breadboard and the side modules can be interfaced using either Teflon tubes or LEGO-type connections. Another primary difference is that, since the culture medium is perfused in this circulation through all modules in a closed loop, a peristaltic pump is utilized to achieve such medium movement rather than the unidirectional reagent injections enabled by pneumatic pressures.

\section{Materials}

\section{Reagents}

! CAUTION All reagents mentioned in this protocol are potentially harmful. It is necessary to use protective clothing (lab coats and gloves) throughout the procedure. Recycle or dispose of solid and liquid wastes according to local or institutional regulations $\triangle$ CRITICAL All reagents should be stored and prepared according to the manufacturers' recommendations.

- Dow SYLGARD 184 silicone encapsulant (Ellsworth, cat. no. 184 SIL ELAST KIT 0.5KG)

- NANO SU-8 negative tone photoresists formulations 2050 (MICRO CHEM, cat. no. SU-8 2050)

- Flow-mix 5min epoxy (Devcon, cat. no. 20445)

- Silver conductive epoxy (MG Chemicals, cat. no. 8330S)

- Potassium iodide (KI, Sigma-Aldrich, cat. no. 221945)

-11-MUA (Sigma-Aldrich, cat. no. 450561)

- SPV (Sigma-Aldrich, cat. no. 85878)

-EDC (Sigma-Aldrich, cat. no. E6383)

- NHS (Sigma-Aldrich, cat. no. 130672)

- Potassium ferricyanide $\left(\mathrm{K}_{3} \mathrm{Fe}(\mathrm{CN})_{6}\right.$, Sigma-Aldrich, cat. no. 702587)

- Sulfuric acid $\left(\mathrm{H}_{2} \mathrm{SO}_{4}\right.$, Sigma-Aldrich, cat. no. 320501)

- $\mathrm{HCl}$ (37\% (vol/vol), Sigma-Aldrich, cat. no. 320331-500ML)

- $\mathrm{NaOH}$ (reagent grade, $\geq 98 \%$ (wt/vol)

- Pellets, anhydrous (Sigma-Aldrich, cat. no.S5881)! CAUTION $\mathrm{H}_{2} \mathrm{SO}_{4}, \mathrm{HCl}$ and $\mathrm{NaOH}$ are corrosive. Personal protective equipment (goggles, face shield, aprons, boots) should be worn when contact is possible.

- Ethanol $\left(\mathrm{C}_{2} \mathrm{H}_{5} \mathrm{OH}, \geq 99.8 \%\right.$ (vol/vol) (GC), Sigma-Aldrich, cat. no. 24102)

- Phosphate-buffered saline (PBS, Gibco cat. no. 14190-144)

- HCM Hepatocyte Culture Medium BulletKit (Lonza, cat. no. CC-3198)

- Penicillin-streptomycin (Gibco, cat. no. 15070063)

- Roswell Park Memorial Institute (RPMI) 1640 medium (Thermo Fisher Scientific, cat. no. 11875093)

- 50× B-27 supplement (Thermo Fisher Scientific, cat. no. 17504044)

- Albumin biotinylated antibody and full-length protein from Albumin ELISA Kit (Abcam cat. no. ab108788, RRID: AB_2876883)

- Glutathione S-transferase-alpha (GST- $\alpha$ ) biotinylated antibody and full-length protein from GST- $\alpha$ ELISA Kit (Abcam, cat. no. ab173835, RRID: AB_2876882)

- Amine group-functionalized creatine kinase-myocardial band (CK-MB) aptamer (OTC biotech, customized) $\triangle$ CRITICAL For reproducibility of these results, the customized aptamer was designed to capture CK-MB1, with a 5'-amine.

- CK-MB full-length protein from CK-MB ELISA kit (Abcam, ab193696; RRID: AB_2876884)

- Acetone (ACS reagent, $\geq 99.5 \%$ (vol/vol), Sigma-Aldrich, cat. no. 179124)

- Isopropyl alcohol (natural, $\geq 98 \%$ (vol/vol), FG), Sigma-Aldrich, cat. no. W292912

- Deionized (DI) water

- Red food coloring (Thermo Fisher Scientific, cat. no. S05371)

- Green food coloring (Thermo Fisher Scientific, cat. no. S05376)

- Milli-Q water (18 M $\Omega \cdot \mathrm{cm}$; Millipore)

- Sodium chloride (NaCl; Adamas, cat. no. 81793B) 
- Square glass substrates $\left(25 \times 25 \mathrm{~mm}^{2}\right)$

- Nitrogen, ultra-high purity, 5.0 grade, size 200 cylinder, CGA-580 (Airgas, cat. no. NI UHP200)

$\triangle$ CRITICAL Nitrogen gas is less prone to expansion compared with other gases, and is absent of moisture, assuring more consistent pressure controls. Nitrogen is nonflammable and does not cause rust or decay.

- SU-8 developer (MicroChem, cat. no. Y020100) ! CAUTION Causes skin and serious eye irritation with some possible allergic skin reactions and respiratory irritation. Wear safety glasses, gloves and face protection. Use inside a ventilated chemical fume hood.

- Trichloro(1H,1H,2H,2H-perfluorooctyl)silane (PFOTS; Sigma-Aldrich, cat. no. 448931) !CAUTION Combustible, causes serious skin burns and eye damage. Wear safety glasses, gloves and face protection. Use inside a ventilated chemical fume hood.

\section{Equipment}

General

- Automated valve controller (custom-assembled according to https://sites.google.com/site/rafa elsmicrofluidicspage/)

- Oven (VWR, cat. no. 89511-406)

- SEM (Zeiss, cat. no. EVO 55)

- Stereo zoom microscope (Laxco, cat. no. MZS1MZ22)

- AFM (Asylum Research, cat. no. Cypher ES)

- Spin coater (Laurell, cat. no. WS-650-23B)

- Benchtop plasma cleaner (Plasma Etch, cat. no. PE-50)

- pH meter (Fisher Scientific, cat. no. 13-636-AB15PC)

- High-capacity vacuum pump (Gast, cat. no. DOA-P504-BM)

\section{Microfabrication}

- Clean room (Class 100)

- Double-sided polyimide cleanroom tape with silicone adhesive (Ultratape, cat. no. 2159)

- Nitrogen spray gun with hose assembly (Airgas, cat. no. GSEKPN-1)

- Rapid thermal processor chamber (AccuThermo, cat. no. AW820)

- Shadow masks for microelectrode fabrication (see Supplementary Data 1)

- Microfluidic designs (see Supplementary Data 1 and Step 11)

- Mask aligner (Karl-Süss, cat. no. MJB-55W)

- Dicing saw machine (DISCO, cat. no. DAD3240)

- E-beam evaporator (Denton, cat. no. EE-4)

- Spin coating workstation (Reynolds Tech, cat. no. SB-3)

- Rapid thermal processor (Modular Process Technology, cat. no. RTP-600xp)

- Microbore tubing (0.012" ID × 0.030" OD; Cole-Parmer, cat. no. EW-06417-11)

- Microbore tubing $\left(0.020^{\prime \prime}\right.$ ID $\times 0.060^{\prime \prime}$ OD; Cole-Parmer, cat. no. EW-06419-01)

- Microbore tubing (0.030" ID × 0.090" OD; Cole-Parmer, cat. no. EW-06419-03)

- Glass microscope slides (Thermo Fisher Scientific, cat. no. 12-550-A3)

- Wafer tweezers (VWR, cat. no. OUTI42LB-6)

- Scalpel (VWR, cat. no. 95039-117)

\section{Hardware and software}

- Standard laptop or desktop running Windows 7 Intel core i5 processor and 4 GB RAM or higher

- EC workstation (CHI Instruments, cat. no. CHI660e)

- MATLAB (MathWorks, https://www.mathworks.com/products/matlab.html)

- Custom-made MATLAB program for controlling the valve system (Electrochemical Biosensing; https://shrikezhang.com/publications/opensource)

- $\mathrm{CH}$ Instruments software (Electrochemical Instrumentation $\mathrm{CH}$ Instruments, https://www. chinstruments.com/downloads.shtml)

\section{Reagent setup}

Self-assembled monolayer (SAM) solution

To make $10 \mathrm{mM}$ SAM solution, dissolve $109.18 \mathrm{mg}$ of MUA in $50 \mathrm{~mL}$ of ethanol. Seal the cap with parafilm to avoid evaporation, and store the solution at $4{ }^{\circ} \mathrm{C}$. The SAM solution can be stored at $4{ }^{\circ} \mathrm{C}$ for $\sim 2$ months. 
Citric buffer solution

To prepare $50 \mathrm{mM}$ citric acid solution, dissolve $0.335 \mathrm{~g}$ of sodium citrate and $0.261 \mathrm{~g}$ of citric acid in $400 \mathrm{~mL}$ of distilled water. Adjust solution to $\mathrm{pH} 4.5$ using $1 \mathrm{M} \mathrm{HCl}$ or $1 \mathrm{M} \mathrm{NaOH}$, and fill the solution to a final volume of $500 \mathrm{~mL}$. The citric buffer can be stored at $4{ }^{\circ} \mathrm{C}$ for at least 6 months.

\section{EDC/NHS solution}

To make equimolar $(25 \mathrm{mM})$ EDC/NHS solution, prepare the $50 \mathrm{mM}$ NHS solution by dissolving $5.75 \mathrm{mg}$ of NHS in $1 \mathrm{~mL}$ of $50 \mathrm{mM}$ citric buffer. Then prepare the $50 \mathrm{mM}$ EDC solution by dissolving $9.55 \mathrm{mg}$ of EDC in $1 \mathrm{~mL}$ of $50 \mathrm{mM}$ citric buffer and mix the two solutions at a 1:1 ratio. The EDC/ NHS solution should be prepared fresh before the experiment.

\section{$\mathrm{K}_{3} \mathrm{Fe}(\mathrm{CN})_{6}$ stock solution}

To make $50 \mathrm{mM} \mathrm{K} \mathrm{K}_{3} \mathrm{Fe}(\mathrm{CN})_{6}$ solution, dissolve $823.15 \mathrm{mg}$ of $\mathrm{K}_{3} \mathrm{Fe}(\mathrm{CN})_{6}$ in $50 \mathrm{~mL}$ of DI water. The $\mathrm{K}_{3} \mathrm{Fe}(\mathrm{CN})_{6}$ solution can be stored at room temperature in the dark for $\sim 2$ months. ! CAUTION $\mathrm{K}_{3} \mathrm{Fe}$ $(\mathrm{CN})_{6}$ is an irritant. Avoid contact with skin and eyes. Avoid breathing dust. Use in a fume hood. $\triangle$ CRITICAL $\mathrm{K}_{3} \mathrm{Fe}(\mathrm{CN})_{6}$ is light sensitive. $\mathrm{K}_{3} \mathrm{Fe}(\mathrm{CN})_{6}$ solution decomposes slowly on standing. Store in dark or opaque bottles and away from light.

\section{SPV stock solution}

To make $10 \mu \mathrm{g} \mathrm{mL}^{-1} \mathrm{SPV}$, prepare SPV solution of $1 \mathrm{mg} \mathrm{mL}^{-1}$ by dissolving $1 \mathrm{mg}$ of SPV in $1 \mathrm{~mL}$ of PBS and then dilute to $10 \mu \mathrm{g} \mathrm{mL} \mathrm{m}^{-1}$ by adding $140 \mu \mathrm{L}$ of the $1 \mathrm{mg} \mathrm{mL}^{-1}$ SPV solution into $13.88 \mathrm{~mL}$ of PBS. Store at $-20^{\circ} \mathrm{C}$ in $2 \mathrm{~mL}$ Eppendorf tubes. The SPV solution can be stored under these conditions for at least 3 months. $\triangle$ CRITICAL Avoid repeated freeze-thaw cycles.

\section{$\mathrm{H}_{2} \mathrm{SO}_{4}$ stock solution}

To prepare $10 \mathrm{mM} \mathrm{H}_{2} \mathrm{SO}_{4}$ solution, mix $500 \mu \mathrm{L}$ of $1 \mathrm{M} \mathrm{H}_{2} \mathrm{SO}_{4}$ with $49.5 \mathrm{~mL}$ of distilled water. The $\mathrm{H}_{2} \mathrm{SO}_{4}$ solution can be stored in a chemical cabinet at room temperature (RT, $18-24{ }^{\circ} \mathrm{C}$ ) for at least 6 months. ! CAUTION Exothermic and hazardous solution. Use in fume hood with protective equipment.

\section{Dilution buffer}

To prepare $2 \mathrm{~mL}$ of $1 \times$ dilution buffer, dilute $200 \mu \mathrm{L}$ of $10 \times$ dilution buffer in the ELISA kit with $1,800 \mu \mathrm{L}$ of distilled water. The dilution buffer should be prepared fresh before the experiment.

\section{Biotinylated antibody solution}

To prepare $10 \mu \mathrm{g} \mathrm{mL}^{-1}$ biotinylated antibody solution, dilute the stock solution in PBS. The biotinylated antibody solution should be prepared fresh before the experiment.

\section{Amine group-functionalized aptamer solution}

To prepare $10 \mu \mathrm{g} \mathrm{mL}^{-1} 5^{\prime}$-end amine group-functionalized aptamer solution, dilute the stock solution in PBS. The amine group-functionalized aptamer solution should be prepared fresh before the experiment.

\section{Washing buffer}

Dilute $10 \times$ washing buffer in ELISA kit to $1 \times$ with distilled water. The washing buffer should be prepared fresh before the experiment.

\section{Hepatocyte culture medium}

Reconstitute according to manufacturer's instructions. The medium should be prepared in aliquots in 50 or $15 \mathrm{~mL}$ conical tubes sealed with parafilm, and can be stored at $4{ }^{\circ} \mathrm{C}$ for $\sim 1 \mathrm{month}$.

\section{Cardiomyocyte culture medium}

Add 10\% (vol/vol) FBS, 1\% (vol/vol) penicillin-streptomycin and 1× B-27 supplement to RPMI 1640 . The medium should be prepared in aliquots in 50 or $15 \mathrm{~mL}$ conical tubes sealed with parafilm and can be stored at $4{ }^{\circ} \mathrm{C}$ for $\sim 1$ month.

\section{Common medium}

Mix hepatocyte culture medium and cardiomyocyte culture medium at 1:1 volume ratio. $\triangle$ CRITICAL Preparation of culture medium and common medium should be adjusted according to the cell type(s) used in the project. 


\section{PDMS}

In a disposable container, load desired amount of elastomer base and curing agent at a 10:1 mass ratio. Using a glass stirring rod, mix the two components thoroughly. Place the container into a vacuum desiccator for $30 \mathrm{~min}$ to remove all bubbles. Pour volume according to molding needs. The PDMS can be stored at $4{ }^{\circ} \mathrm{C}$ for $\sim 1$ month or prepared fresh before each fabrication.

\section{Equipment setup}

Setup of the EC cell

In this protocol, all experiments are carried out by the three-electrode system fabricated on the microelectrodes $\mathrm{Au}$ WE, Ag RE and Au CE. We have used cyclic voltammetry (CV) for cleaning purposes and EIS for signal measurements. Randles circuit, $R_{\mathrm{s}}\left(C_{\mathrm{dl}}\left[R_{\mathrm{ct}} W\right]\right)$ with solution resistance, $R_{\mathrm{s}}$, double-layer capacitance, $C_{\mathrm{dl}}$, Warburg element, $W$, and charge transfer resistance, $R_{\mathrm{ct}}$, are used to fit the EIS data ${ }^{38}$.

\section{Procedure}

\section{Microelectrode fabrication Timing $5 \mathrm{~h}$}

1 Wash square glass substrates in acetone, and dry with compressed air gun in a cleanroom.

2 Attach the first shadow mask ( $0.25 \mathrm{~mm}$ in thickness) for WE and CE to the glass substrates using double-sided tape, and place them in the electron-beam evaporator.

3 Wait until the chamber pressure reaches at least $10^{-6}$ level. This step takes $\sim 45-60 \mathrm{~min}$.

4 Deposit $20 \mathrm{~nm}$ Ti layer, $20 \mathrm{~nm}$ Pd layer and $500 \mathrm{~nm}$ Au layers for both WE and CE (Fig. 1a).

5 Attach the second shadow mask for RE on the Au-deposited glass substrates using double-sided tape, and place in the electron-beam evaporator.

$\triangle$ CRITICAL STEP Check all the microelectrodes one by one to make sure there is good alignment for all the REs and WEs/CEs.

6 Wait until the chamber pressure reaches at least $10^{-6}$ level. This step takes $\sim 45-60 \mathrm{~min}$.

7 Deposit $20 \mathrm{~nm}$ Ti layer, $20 \mathrm{~nm}$ Pd layer and $500 \mathrm{~nm} \mathrm{Ag} \mathrm{layers} \mathrm{for} \mathrm{RE} \mathrm{(Fig.} \mathrm{1a).}$

8 After the deposition step, remove the samples from the electron-beam evaporator.

9 Anneal the fabricated microelectrodes in a nitrogen atmosphere using a rapid thermal processor chamber $\left(300{ }^{\circ} \mathrm{C}, 2 \mathrm{~h}\right)$.

! CAUTION Wait until temperature reaches RT before removing the samples.

$\triangle$ CRITICAL STEP Handle the microelectrodes carefully, making sure they do not scratch.

10 Cut the fabricated microelectrode substrates $\left(25 \times 25 \mathrm{~mm}^{2}\right)$ using a dicing saw machine.

DAUSE POINT The microelectrodes can be stored indefinitely at RT away from dust.

\section{Photolithographic fabrication of microfluidic controlling devices Timing $18 \mathrm{~h}$}

$\triangle$ CRITICAL Steps 13-31 should be carried out inside a clean room facility. Dust and light exposure to resin and related materials decrease microfabrication precisions. Exposure of SU-8 to white light causes partial or complete crosslinking, disrupting the resin's optical transparency.

11 Use AutoCad to design the following masks: (i) the fluidic layer of the breadboard, (ii) the valve layer of the breadboard, (iii) the fluidic layer of the EC detection chip and (iv) the valve layer of the EC detection chip. See Supplementary Data 1 for our example designs.

12 Print the photomasks through a commercial source (e.g., CAD/Art Services, https://www.outputcity.com/). The photomasks are in the form of transparent polymer films, where the patterns are left transparent.

13 Cut the photomasks individually.

$\triangle$ CRITICAL STEP Handle photomasks with gloves at all times. Skin grease and impurities can diffract light during photolithography.

14 Place a $4^{\prime \prime}$ silicon wafer on the spinner chuck of a spin coater and apply nitrogen vacuum.

15 To clean the wafer surface, spin the wafer at 3,000 r.p.m. for $1 \mathrm{~min}$ at room temperature and in sequence apply acetone, methanol and isopropyl alcohol.

16 Remove solvents by placing the wafer on a hot plate at $200{ }^{\circ} \mathrm{C}$ for $10 \mathrm{~min}$.

17 Place wafer on a clean and even surface, and allow it to cool for $2 \mathrm{~min}$.

! CAUTION Handle with wafer tweezers to avoid melting of nitrile gloves and/or skin burns.

18 On the center of the wafer, pour $1 \mathrm{~mL}$ of SU-8 2050 photoresist per inch of surface.

$\triangle$ CRITICAL STEP Pour photoresist slowly and carefully to prevent the formation of air bubbles. 
19 With the wafer tweezers, pick up the wafer and place it centered back onto the spin coater. Fix it in place by turning on the vacuum. Let it sit for 5 min.

20 Set up the spin coater and run the wafer according to the following coating conditions:

- Acceleration: 100 r.p.m. s ${ }^{-1}$

- Spread: 500 r.p.m. for $15 \mathrm{~s}$

- Acceleration: 200 r.p.m. s ${ }^{-1}$

- Spin cycle: 1,700 r.p.m. for $30 \mathrm{~s}$

- Break cycle: 500 r.p.m. for $10 \mathrm{~s}$

21 Carefully transfer the covered wafer into a leveled hot plate at $65^{\circ} \mathrm{C}$ for $5 \mathrm{~min}$, for prebake.

22 Cautiously transfer the prebaked wafer into an oven at $95{ }^{\circ} \mathrm{C}$ for $20 \mathrm{~min}$, for soft bake.

23 Place the wafer on an even surface at RT to let cool for $10 \mathrm{~min}$.

24 Prepare mask aligner by loading the photomask (either the corresponding valve layer or fluidic layer mask) and the wafer.

25 Set the mask and the wafer into conformal contact, and expose to UV light for $26 \mathrm{~s}$ at $9.5 \mathrm{~mW} \mathrm{~cm}^{-2}$.

26 Immediately, perform a postexposure bake by placing the exposed wafer on the hot plate at $65^{\circ} \mathrm{C}$ for $4 \mathrm{~min}$, and then into an oven for $9 \mathrm{~min}$ at $95^{\circ} \mathrm{C}$.

27 Develop the wafer by immersing the wafer in SU-8 developer for $15 \mathrm{~min}$.

28 Pick up the wafer with tweezers, and rinse off undeveloped resin by spraying with SU-8 developer.

29 Stop the developer reaction by spraying the wafer with isopropyl alcohol.

30 Dry the wafer with pressurized, filtered nitrogen gas.

31 In a glass container that can fit the wafer and a small liquid container, place the wafer upside down, held on the edges. In the liquid container, place three drops $(\sim 60 \mu \mathrm{L})$ of PFOTS. Leave for overnight silanization through chemical vapor deposition.

! CAUTION Wear safety glasses, gloves and face protection at all times when handling silane. Perform salinization under a ventilated chemical fume hood.

DAUSE POINT Store the silanized master wafers at RT until needed. The master molds can be stored indefinitely away from dust.

\section{PDMS membrane fabrication Timing $1.5 \mathrm{~h}$}

32 Place the lid of a $100 \mathrm{~mm}$ Petri dish facing down in the spin-coater vacuum chuck. Pour $1 \mathrm{~g}$ of degassed 10:1 PDMS pre-polymer.

33 Set up the spin coater and run the Petri dish according to the following coating conditions:

- Acceleration: 300 r.p.m. s ${ }^{-1}$

- Spread: 500 r.p.m. for $10 \mathrm{~s}$

- Acceleration: 500 r.p.m. s ${ }^{-1}$

- Spin cycle: 1,000 r.p.m. for $30 \mathrm{~s}$

- Break cycle: 300 r.p.m. for $10 \mathrm{~s}$

34 Cover the surface with the bottom of the Petri dish facing down, to protect the newly generated membrane.

$\triangle$ CRITICAL STEP When placing the bottom cover and handling the coated lid, avoid any disturbance that might disrupt the PDMS layer.

35 Cure PDMS in an oven at $80^{\circ} \mathrm{C}$ for $1 \mathrm{~h}$.

$\triangle$ CRITICAL STEP Make sure the coated Petri dish is level inside the oven during curing. Avoid stacking the Petri dishes on top of each other.

36 Remove the coated Petri dish from the oven, and allow to cool at RT for $20 \mathrm{~min}$.

DAUSE POINT Store PDMS-coated dishes covered by the bottoms of the Petri dishes. Do not remove the covers from the membranes until ready to use. The PDMS membranes can be stored indefinitely at RT.

\section{Fluidic layer fabrication Timing $\mathbf{3}$ h}

37 Use pressurized nitrogen to blow dust particles from the silicone master wafer mold of the fluidic layer from Step 31.

38 Using double-sided tape, attach the corresponding silicone master of the fluidic layer to enough aluminum foil to encase it in a circle of $5 \mathrm{~mm}$-high walls.

39 Pour enough degassed 10:1 PDMS pre-polymer onto the wafer to generate a $3 \mathrm{~mm}$-thick PDMS layer.

40 Place the PDMS pre-polymer-covered wafer in a vacuum chamber for $30 \mathrm{~min}$, for further degassing. 
41 Transfer the wafer to an oven, and cure PDMS at $80^{\circ} \mathrm{C}$ for at least $2 \mathrm{~h}$.

$\triangle$ CRITICAL STEP Make sure the wafer is level inside the oven during curing.

42 Remove from the oven, and allow it to cool at RT for $20 \mathrm{~min}$.

43 By using a scalpel, carefully remove the negatively casted cured PDMS from the wafer. ! CAUTION Be careful when using the scalpel, to avoid scratches to the wafer and/or user injury. DAUSE POINT Store the PDMS-cast fluidic layer, protecting it from dust by placing it inside a clean Petri dish. The PDMS fluidic layer can be stored indefinitely at RT.

\section{Hemicylindrical microchannel fabrication Timing $16 \mathrm{~h}$}

44 Remove the protective lid from a fabricated membrane (from Step 35), and place the uncovered membrane inside the plasma chamber.

45 Remove the PDMS replica of the fluidic layer fabricated in Step 42 from the clean Petri dish. Use a $20 \mathrm{G}$ punch to punch out a single inlet of the corresponding valve layer.

$\triangle$ CRITICAL STEP Make sure the PDMS 'worm' is fully removed and no lacerations occur in the PDMS. This is the port for the generation of a tight negative pressure that will homogeneously deform the thin PDMS membrane, and a smooth and tight grip of the hole is needed (Fig. 3b).

46 Place the negative channels facing up inside the plasma chamber.

47 Treat simultaneously both the PDMS membrane and the PDMS replica with plasma for $\sim 60-90 \mathrm{~s}$.

48 Place the negative feature side of the PDMS replica facing the membrane. Bond the PDMS replica to the membrane immediately following plasma treatment.

49 Place the bonded piece into an oven at $80{ }^{\circ} \mathrm{C}$ for another $30 \mathrm{~min}$ to secure bonding.

$\triangle$ CRITICAL STEP Handle all materials with gloves to avoid oil or dust contamination. We also recommend the use of surgical masks to avoid any particle contamination of the surfaces.

50 Place a $127 \times 102 \mathrm{~mm}^{2}$ glass slide on a spin coater and apply vacuum.

51 On the center of the glass slide, pour $1 \mathrm{~mL}$ of SU-8 2050 photoresist per inch of surface.

52 Set up the spin coater, and run the glass slide according to the following coating conditions:

- Acceleration: 100 r.p.m. s ${ }^{-1}$

- Spread: 500 r.p.m. for $15 \mathrm{~s}$

- Acceleration: 200 r.p.m. s ${ }^{-1}$

- Spin cycle: 1,700 r.p.m. for $30 \mathrm{~s}$

- Break cycle: 500 r.p.m. for $10 \mathrm{~s}$

53 Carefully transfer the SU-8-covered glass slide onto a level surface.

$\triangle$ CRITICAL STEP When handling the SU-8-covered glass slide, avoid any disturbance that might disrupt the SU-8 layer.

54 Remove the bonded PDMS from the oven, and allow it to cool at RT for $20 \mathrm{~min}$.

55 By using a scalpel, carefully cut the edges of the bonded PDMS membrane and replica from the Petri dish lid.

! CAUTION Be careful when using the scalpel, to avoid scratches to the PDMS or user injury.

56 Insert a $27 \mathrm{G}$ metal connector into the inlet on the featureless side of the slab.

57 Connect the metal connector into Teflon tubing, and further extend the connection to a vacuum pump.

58 Set vacuum at $10 \mathrm{mmHg}$, and apply negative pressure by turning on the vacuum.

$\triangle$ CRITICAL STEP At this point, all the negative microchannels should have the membrane deformed into the grooves (Fig. 3b). Make sure all tubing and connectors are tightly sealed into the PDMS to avoid uneven distribution of vacuum in the microchannels.

? TROUBLESHOOTING

59 With the pump on, place the edge of the PDMS-bonded slab in a $45^{\circ}$ contact with the SU-8 cover glass slide. Make sure the negatively bent membrane features are facing the SU- 8 .

60 In a steady and single movement, place the slab in full contact with the SU-8. Let the arrangement sit for $10 \mathrm{~min}$, allowing SU-8 to fill the entirety of the grooves.

$\triangle$ CRITICAL STEP Avoid the formation of bubbles or the application of forces that will spread the SU-8 unevenly.

61 Place the PDMS/SU-8 arrangement under UV light at $850 \mathrm{~mW}$ for $3 \mathrm{~min}$ at a $5 \mathrm{~mm}$ distance from the glass side.

62 Remove the arrangement from the UV light. Turn off the vacuum, and carefully remove the PDMS slab from the baked SU-8 positive mold. 
63 In a glass container that can fit the secondary mold with hemicylindrical features and a small liquid container, place the mold upside down, held on the edges. In the liquid container, place three drops $(\sim 60 \mu \mathrm{L})$ of PFOTS. Leave for overnight silanization through chemical vapor deposition.

! CAUTION Wear safety glasses, gloves and face protection at all times when handling silane. Perform salinization under a ventilated chemical fume hood.

64 Repeat Steps 36-42 to generate the PDMS replica of the fluidic layer containing hemicylindrical microchannels.

65 Use a scalpel to cut off the out-of-plane edges of the fluidic layer slab to facilitate its bonding with the valve layer.

DAUSE POINT Store the PDMS-cast valve layer containing hemicylindrical microchannels, protecting it from dust by placing it inside a clean Petri dish. The PDMS fluidic layer with hemicylindrical microchannels can be stored indefinitely at RT.

\section{EC detection chip assembly Timing $20 \mathrm{~h}$}

$\triangle$ CRITICAL STEP Steps 65-86 should be carried out utilizing gloves to handle all materials to avoid oil or dust contamination. We also recommend the use of surgical masks to avoid any particle contamination of the surfaces.

66 Use pressurized nitrogen to blow dust particles away from the silicone master wafer mold for the valve layer of the EC detection chip.

67 Repeat Steps 36-42 to generate the PDMS replica of the valve layer.

68 Clean the cured PDMS replica of the valve layer from dust and impurities by firmly placing Scotch tape on its surface, and in one single and rapid movement peel it off.

69 Use a $20 \mathrm{G}$ punch to punch out the inlets of the valve layer.

$\triangle$ CRITICAL STEP Make sure the PDMS 'worms' are fully removed and no lacerations occur in the PDMS. These are the pressure ports of the system, and a smooth and tight grip of the holes is needed. ! CAUTION When using the punch, be careful to avoid damage to the PDMS or user injury.

70 Place the PDMS valve layer replica into the plasma chamber, with the microchannels facing up.

71 Remove the protective lid from a fabricated PDMS membrane, and place the uncovered membrane inside the plasma chamber.

72 Treat both PDMS membrane and the replica simultaneously with nitrogen plasma for 60-90 s.

73 Bond the PDMS replica to the membrane, by placing the feature side of the PDMS slab in contact with the membrane immediately after plasma treatment.

74 Place the bonded piece into an oven at $80^{\circ} \mathrm{C}$ for $30 \mathrm{~min}$ to further secure bonding.

75 Remove the bonded PDMS from the oven, and allow it to cool at RT for $20 \mathrm{~min}$.

76 By using a scalpel, carefully cut the edges of the bonded membrane and PDMS slab, and peel the piece from the Petri dish lid.

$\triangle$ CRITICAL STEP Remove the bonded membrane slowly and carefully to avoid fissure or wreckage of the membrane.

77 With a scalpel, evenly cut off the edges of the valve layer slab to facilitate its bonding with the fluidic layer.

! CAUTION Be careful when using the scalpel, to avoid scratches to the PDMS or self-harm.

78 Use a 20G punch to punch out the inlets and outlets through the PDMS replica of the valve layer, in marked positions (red dots in Fig. 3a) corresponding to the microchannel inlets in the fluidic layer. $\triangle$ CRITICAL STEP Make sure the PDMS 'worms' are fully removed and no lacerations occur in the PDMS. These are the flow ports of the system, and a smooth and tight grip of the holes is needed.

79 Use a $3 \mathrm{~mm}$ punch to punch out the detection chambers through a PDMS replica of the fluidic layer, prepared in Step 64 (Fig. 3a).

! CAUTION Be careful when using the punch, to avoid scratches to the PDMS or user injury.

80 Clean the PDMS replica of the fluidic layer and the valve layer from dust and impurities by firmly placing Scotch tape on the surface, and in one single and rapid movement peeling it off.

81 Place both the PDMS replica of the fluidic layer and the membrane-bond valve layer with feature sides facing up in the plasma chamber.

82 Treat both layers with nitrogen plasma simultaneously for 60-90 s.

83 Remove the plasma-activated PDMS slabs, and fully cover both with 200 proof ethanol.

84 Place the fluidic layer with hemicylindrical channels on top of the membrane-bond valve layer, by facing the negative feature side of the fluidic layer with the membrane side of the valve layer (Fig. 3a). 
$\triangle$ CRITICAL STEP Although the surface-active groups on PDMS can be maintained in ethanol for extended periods of time, perform the bonding as soon as possible to avoid any possible deterioration of the functional groups.

85 With the help of a stereoscope, as needed, align all the valve points with their corresponding microchannels.

$\triangle$ CRITICAL When the two layers are aligned, make sure to hold them for enough time to allow evaporation of the excess ethanol, avoiding additional sliding between the two layers.

86 Carefully transfer the aligned chip assembly into a Petri dish with a Kimwipe placed at the bottom, and cover with the lid. Leave the assembly at RT until complete evaporation of ethanol. Then, place it into an oven at $80{ }^{\circ} \mathrm{C}$ overnight.

87 Remove the bonded PDMS from the oven, and allow it to cool at RT for $20 \mathrm{~min}$.

- PAUSE POINT Store and protect the EC detection chips from dust by placing them inside a Petri dish until use. The EC detection chips can be stored indefinitely at RT.

\section{EC detection chip and sensor incorporation Timing $1 \mathrm{~h}$}

$\Delta$ CRITICAL STEP Steps 87-95 should be carried out utilizing gloves to handle all materials to avoid oil or dust contamination. We also recommend the use of surgical masks to avoid any particle

contamination of the surfaces.

88 Remove an assembled EC detection chip from the Petri dish. Clean dust and impurities from the surface using Scotch tape.

89 Clean three microelectrodes from dust and impurities by blowing with a compressed nitrogen gun.

90 Place the microelectrodes facing upwards, and the EC detection chip with bottom facing up, into the plasma chamber.

$\triangle$ CRITICAL STEP The open detection chambers in the fluidic layer of the PDMS chip should face up.

91 Treat the microelectrodes and the chip simultaneously with the nitrogen plasma for 30-60 s.

$\triangle$ CRITICAL STEP Oxidation of the RE, manifested by turning black, can cause corrosive resistance. We recommend terminating the microelectrode if this happens.

92 Bond the microelectrodes to the PDMS-based EC sensing chip, with the detection areas aligning with the corresponding chambers (Fig. 1b \& 3a).

93 Use silver epoxy to ensure electrical contact in connecting the three leads of each microelectrode and individual copper wires. Place in an oven at $80{ }^{\circ} \mathrm{C}$ for $30 \mathrm{~min}$.

$\triangle$ CRITICAL STEP After curing, check electrical connections by a multimeter.

94 Use 5 min epoxy to further secure the connection points between the copper wires and the microelectrode leads to prevent any breakage during usage.

95 Use 5 min epoxy to cover also the connection between the microelectrodes and the PDMS chip to prevent any leakage during usage.

96 Insert Teflon tubing into the valve and fluidic layer inlets and outlets for gas and liquid flows. With 5-min epoxy, secure all tubing/PDMS connections to prevent any leakage during usage.

! CAUTION Use personal protective equipment throughout the procedure. Epoxy can be an irritant to skin and damaging to clothes.

$\triangle$ CRITICAL STEP Use a P-10 microtip to place the epoxy around the tubing, to avoid disrupting the tubing or introducing extra epoxy.

PAUSE POINT Place assembled EC detection chips individually in Petri dishes and keep away from dust at RT until ready for integration. The assembled EC detection chips can be stored indefinitely under these conditions, avoiding humidity.

\section{Reservoir fabrication Timing $\mathbf{1 . 5} \mathbf{h}$}

97 With an $18 \mathrm{G}$ needle, punch two holes into the lid of a cryogenic vial, or a conical tube, depending on the amount of reagent needed.

98 Use a scalpel to cut enough Teflon tubing. ! CAUTION Be careful when using the scalpel and needle, to avoid vial scratching or user injury.

99 Through one of the punched holes, insert one Teflon tube all the way into the bottom of the vial (outlet).

100 Through the second hole, partially insert a second Teflon tube to just underneath the lid (inlet) (Fig. 3c-ii).

101 Use 5 min epoxy to fix and seal the tubing in place on the vial lid. 
102 Place the vial in a vertical position in an assembled reservoir holder. Let epoxy completely cure.

103 Inside a laminar flow hood, wash both interiors and exteriors of the reservoir and the tubing using $70 \%$ (vol/vol) ethanol followed by rinsing with sterile PBS.

104 Place the reservoir under UV light, and further sterilize for $30 \mathrm{~min}$.

DAUSE POINT The sterile reservoirs can be stored at RT indefinitely until ready for integration. If the reservoirs are used for reagents and have no direct contact with cell culture, they might not need to be sterilized.

\section{Breadboard assembly Timing $20 \mathrm{~h}$}

$\triangle$ CRITICAL STEP Steps 104-108 should be carried out utilizing gloves to handle all materials to avoid oil or dust contamination. We also recommend the use of surgical masks to avoid any particle

contamination of the surfaces.

105 Use pressurized nitrogen to blow dust particles away from the silicone master molds of the breadboard valve layer and hemicylindrical flow layer.

106 Repeat Steps 36-42 to generate the PDMS replicas of both layers.

107 Use a 20G punch to punch out the inlets of the valve layer, and the inlets and outlets of the flow layer.

$\triangle$ CRITICAL STEP Make sure the PDMS 'worms' are fully removed and no lacerations occur in the PDMS. These are the ports for gas and fluid flows, and a smooth and tight grip of the holes is needed.

! CAUTION Be careful when using the needle, to avoid damage to the PDMS or user injury.

108 Repeat Steps 69-86 to assemble the membrane and the breadboard, except for Step 78, as the breadboard has no detection chambers (Fig. $4 \mathrm{a}$ ).

109 Repeat Step 95 to connect the tubing to all inlets and outlets.

DAUSE POINT Place assembled breadboards individually in Petri dishes, and keep away from dust at RT until ready for integration. The assembled breadboards can be stored indefinitely under these conditions.

\section{Automation operational system preparation Timing $1.5 \mathrm{~h}$}

110 Turn on the nitrogen gas flows and regulators for valve and fluid controls.

111 Turn on WAGO controller for Festo solenoid valves, and connect the system to the computer running the custom-coded MATLAB program (https://shrikezhang.com/publications/opensource or ref. ${ }^{13}$ ).

$\triangle$ CRITICAL STEP Steps 111-120 should be carried out utilizing gloves to handle all materials to avoid oil, dust or biological contamination. We also recommend the use of surgical masks to avoid any further contamination.

112 Remove one EC sensing chip and one breadboard from their respective Petri dishes.

113 Carefully fill tubing connecting to all the valves with food dye prepared in water. Food dye is not a necessity, although it will aid the visualization.

114 Connect the solenoid system to gas flow control. Individually connect each solenoid to its corresponding Teflon tubing linked to the valves on the chips, through metal connectors.

115 Allow for the valve microchannels on the chips to be filled by opening the solenoids and applying 5-15 PSI of gas pressure for $\sim 30 \mathrm{~min}$.

! CAUTION Constantly monitor each valve as it gets filled, since overly high pressures can burst the membrane layers.

? TROUBLESHOOTING

116 On the EC sensing chip, leave the solenoids open to keep the valves on the chip closed to seal the flow microchannels (Fig. 3c-iii).

117 Fill the reservoirs with their corresponding reagents (Fig. 3d-f).

118 Individually connect the remaining solenoids to their corresponding reservoirs through the inlet tubing.

119 Set the pneumatic pressure at 1-5 PSI to drive each specific reagent through the reservoir's outlet tubing (Fig. 3c-ii).

$\triangle$ CRITICAL STEP Constantly monitor each reservoir as the solution flows through the outlet tubing. Stop each solenoid as the corresponding solution reaches the end of the tubing (Fig. 3c-iv). Steps $117-118$ can also be done manually as needed. 
120 Connect each outlet tubing on the reservoir to its corresponding flow layer inlet on the chip through a metal connector.

121 Turn the solenoids off to open the valves on the chip, and reinitiate the pneumatic pressures driving the reagents into their corresponding flow microchannels, until all the microchannels are filled with their desired reagents and are bubble free.

$\triangle$ CRITICAL STEP Each flow microchannel should be individually filled and monitored as it gets filled, to avoid contamination of reagents across the microchannels. Also, turning off the solenoids controlling the valves on the chip and initiating the pneumatic pressures driving the reagent flows can be programmed a few seconds apart in the MATLAB codes, to avoid abrupt changes in pressures during operations that might disturb the flows.

PAUSE POINT The EC sensing chip and breadboard are now ready to integrate with the microbioreactors. Ideally these individual components should be used immediately following preparation, but if necessary they can be stored in a parafilm-sealed humidified box at $4{ }^{\circ} \mathrm{C}$ for up to 1 month.

\section{Microbioreactor and breadboard fluidic integration Timing $1.5 \mathrm{~h}$}

$\triangle$ CRITICAL STEP Steps 121-136 should be carried out utilizing gloves to handle all materials to avoid biological contamination. We also recommend the use of surgical masks to avoid any further contamination.

122 Insert the inlet tubing of a sterile reservoir into an $\mathrm{MP}^{2}$ peripump PVC tube.

123 Place the $\mathrm{MP}^{2}$ peripump PVC tubing into one of the grooves of an ESI MP ${ }^{2}$ peristaltic pump.

124 Connect the outlet tubing of the reservoir with a metal connector into tubing inserted into the medium inlet on the breadboard (Fig. $4 \mathrm{~b}-\mathrm{d}$ ).

125 Fill the reservoir with desired amount of desired medium (e.g., $5 \mathrm{~mL}$ ), and tightly seal it.

126 Set up the peristaltic pump at desired flow rate (e.g., $200 \mu \mathrm{L} \mathrm{h}^{-1}$ ). The increased pressure inside the reservoir will be released by the flow of medium through the reservoir outlet tubing into the flow microchannel of the breadboard.

$\triangle$ CRITICAL STEP Medium should flow through the tubing and microchannels smoothly with no back flow.

\section{? TROUBLESHOOTING}

127 Stop the peristaltic pump once medium reaches end of the tubing inserted in the organ-1 inlet on the breadboard.

128 Remove one assembled microbioreactor from the incubator.

129 Connect the tubing coming from organ-1 inlet to the inlet tubing of the microbioreactor through a metal connector (Fig. $4 b-d$ ).

130 Connect the outlet tubing of the microbioreactor to the organ-1 outlet tubing on the breadboard.

131 Start the micropump again.

$\triangle$ CRITICAL STEP Medium should flow through the tubing, microbioreactor and microchannels smoothly with no back flow.

132 Stop the peristaltic pump once medium reaches the end of the tubing inserted in the EC sensor inlet on the breadboard.

133 Gather one assembled EC sensing chip prepared in Step 120.

134 Connect the tubing inserted at the microbioreactor inlet in the EC sensing chip (Fig. $3 \mathrm{~d}-\mathrm{f}$ ) to the corresponding tubing coming from the EC sensor-inlet on the breadboard with a metal connector (Fig. 4b-d). Repeat with the outlet tubing.

135 Re-start the peristaltic pump.

$\triangle$ CRITICAL STEP All the valves of the EC sensing chip should remain closed except for the two controlling the microbioreactor flow in/out. Medium should flow smoothly through the tubing and the desired microchannels in the EC detection chip and microbioreactors with no back flow.

136 Repeat Steps 126-130 to connect the second microbioreactor through the organ-2 inlet/outlet to the breadboard flow.

137 Connect the outlet tubing of the medium reservoir to the free side of the $\mathrm{MP}^{2}$ peripump PVC tubing with a metal connector.

- PAUSE POINT The EC sensing chip, breadboard and bioreactors are now sharing a constant medium stream, and the system is now ready to run EC functionalization and detection of the released biomarkers. Once assembled, the integral system should be used immediately. 


\section{Functionalization of the microfluidic EC sensing chip Timing $6 \mathrm{~h}$}

138 Flow PBS from the main microchannel for $7 \mathrm{~min}$ at $1.0 \mathrm{~mL} \mathrm{~h}^{-1}$.

139 Perform the first step of EC cleaning by flowing the $10 \mathrm{mM} \mathrm{H}_{2} \mathrm{SO}_{4}$ solution for $7 \mathrm{~min}$ at $1.0 \mathrm{~mL} \mathrm{~h}^{-1}$ followed by additional $7 \mathrm{~min}$ flow under the $\mathrm{CV}$ potential sweep with the $\mathrm{CV}$ parameters of:

\begin{tabular}{ll} 
Parameter & Value \\
\hline Initial E (V) & 0.0 \\
High E (V) & 1.8 \\
Low E (V) & 0.0 \\
Final E (V) & 0.0 \\
Initial scan polarity & Positive \\
Scan rate $\left(\mathrm{V} \mathrm{s}^{-1}\right)$ & 0.1 \\
Sweep segments & 8 \\
Sample interval $(\mathrm{V})$ & 0.001 \\
Quiet time $(\mathrm{s})$ & 2 \\
Sensitivity $\left(\mathrm{A} \mathrm{V}^{-1}\right)$ & $1.0 \times 10^{-0.002}$ \\
Communication port speed & Fast \\
Line frequency & $60 \mathrm{~Hz}$ \\
Potential axis & Positive left
\end{tabular}

$\triangle$ CRITICAL STEP Do not stop the $\mathrm{H}_{2} \mathrm{SO}_{4}$ flow during EC cleaning.

140 Open the PBS valve, and flow PBS from the main microchannel for $7 \mathrm{~min}$ at $1.0 \mathrm{~mL} \mathrm{~h}^{-1}$.

141 Perform the second step of EC cleaning by flowing the $50 \mathrm{mM} \mathrm{K}_{3} \mathrm{Fe}(\mathrm{CN})_{6}$ solution for $7 \mathrm{~min}$ at $1.0 \mathrm{~mL} \mathrm{~h}^{-1}$ followed by additional $7 \mathrm{~min}$ flow under the $\mathrm{CV}$ potential sweep with the $\mathrm{CV}$ parameters of:

\begin{tabular}{ll} 
Parameter & Value \\
\hline Initial E (V) & 1.0 \\
High E (V) & 1.2 \\
Low E (V) & -1.2 \\
Final E (V) & 0.0 \\
Initial scan polarity & Negative \\
Scan rate $\left(\mathrm{V} \mathrm{s}^{-1}\right)$ & 0.2 \\
Sweep segments & 6 \\
Sample interval $(\mathrm{V})$ & 0.002 \\
Quiet time $(\mathrm{s})$ & 0 \\
Sensitivity $\left(\mathrm{A} \mathrm{V} \mathrm{V}^{-1}\right)$ & $1.0 \times 10^{-0.004}$ \\
Communication port speed & Fast \\
Line frequency & $60 \mathrm{~Hz}$ \\
Potential axis & Positive left
\end{tabular}

$\triangle$ CRITICAL STEP Do not stop the $\mathrm{K}_{3} \mathrm{Fe}(\mathrm{CN})_{6}$ flow during EC cleaning. 142 Measure the bare microelectrode signal via $\mathrm{CV}$ with the parameters as follows:

\begin{tabular}{lc} 
Parameter & Value \\
\hline Initial E (V) & 0.0 \\
High E (V) & 0.6 \\
Low E (V) & -0.6 \\
Final E (V) & 0.0 \\
& Table continued
\end{tabular}




\begin{tabular}{ll} 
(continued) & \\
Parameter & Value \\
\hline Initial scan polarity & Negative \\
Scan rate $\left(\mathrm{V} \mathrm{s}^{-1}\right)$ & 0.075 \\
Sweep segments & 6 \\
Sample interval $(\mathrm{V})$ & 0.001 \\
Quiet time (s) & 0 \\
Sensitivity $\left(\mathrm{A} \mathrm{V}^{-1}\right)$ & $1.0 \times 10^{-0.004}$ \\
Communication port speed & Fast \\
Line frequency & $60 \mathrm{~Hz}$ \\
Potential axis & Positive left \\
&
\end{tabular}

$\triangle$ CRITICAL STEP Stop the $\mathrm{K}_{3} \mathrm{Fe}(\mathrm{CN})_{6}$ flow, and close the inlet and outlet valves of the detection chamber before starting the $\mathrm{CV}$ procedure.

143 Measure the bare microelectrode signal via EIS with the parameters as follows:

\begin{tabular}{ll} 
Parameter & Value \\
\hline Initial E (V) & 0.0 \\
High frequency $(\mathrm{Hz})$ & 100,000 \\
Low frequency $(\mathrm{Hz})$ & 0.1 \\
Amplitude $(\mathrm{V})$ & 0.05 \\
Quiet time $(\mathrm{s})$ & 0 \\
Sensitivity $\left(\mathrm{A} \mathrm{V}^{-1}\right)$ & $1.0 \times 10^{-0.004}$ \\
Bias DC current during run & $<1 \mathrm{~Hz}$
\end{tabular}

144 Open the ethanol valve, and flow absolute ethanol for $7 \mathrm{~min}$ at $1 \mathrm{~mL} \mathrm{~h}^{-1}$.

$\triangle$ CRITICAL STEP Bubble formation might occur in this step because of the change in polarity of reagents from $\mathrm{K}_{3} \mathrm{Fe}(\mathrm{CN})_{6}$ solution to ethanol flowing through the main microchannel. If bubble formation occurs, increase the flow time for ethanol until bubbles disappear.

145 Open the SAM valve, and flow $10 \mathrm{mM}$ SAM for $7 \mathrm{~min}$ at $1 \mathrm{~mL} \mathrm{~h}^{-1}$.

$\triangle$ CRITICAL STEP Close all other valves except the SAM valve to ensure that the detection chamber is only filled with SAM without any possible contamination by other reagents. While this is true for all operations in this section, it is particularly important for the SAM functionalization step.

146 Incubate SAM for $1 \mathrm{~h}$ at RT.

147 Open the ethanol valve, and flow absolute ethanol for $7 \mathrm{~min}$ at $1 \mathrm{~mL} \mathrm{~h}^{-1}$.

148 Open the PBS valve, and flow PBS from the main microchannel for $7 \mathrm{~min}$ at $1.0 \mathrm{~mL} \mathrm{~h}^{-1}$.

$\triangle$ CRITICAL STEP Bubble formation might occur in this step because of the change in polarity of reagents from ethanol to PBS flowing through the main microchannel. If bubble formation occurs, increase the flow time for PBS until bubbles disappear.

149 Open the EDC/NHS valve, and flow $25 \mathrm{mM}$ equimolar solution of EDC/NHS for $7 \mathrm{~min}$ at $1.0 \mathrm{~mL} \mathrm{~h}^{-1}$.

150 Incubate EDC/NHS solution for $30 \mathrm{~min}$ at RT.

151 Open the SPV valve, and flow $10 \mu \mathrm{g} \mathrm{mL} \mathrm{m}^{-1} \mathrm{SPV}$ for $7 \mathrm{~min}$ at $1.0 \mathrm{~mL} \mathrm{~h}^{-1}$.

152 Incubate SPV for $1 \mathrm{~h}$ at RT.

153 Open the PBS valve, and flow PBS from the main microchannel for $7 \mathrm{~min}$ at $1.0 \mathrm{~mL} \mathrm{~h}^{-1}$.

154 Open the $\mathrm{K}_{3} \mathrm{Fe}(\mathrm{CN})_{6}$ valve, and flow $50 \mathrm{mM} \mathrm{K} \mathrm{K}_{3} \mathrm{Fe}(\mathrm{CN})_{6}$ solution for $7 \mathrm{~min}$ at $1.0 \mathrm{~mL} \mathrm{~h}^{-1}$.

155 Measure the SPV-modified microelectrode signal via EIS with the parameters as listed in Step 142.

$\triangle$ CRITICAL STEP Stop the $\mathrm{K}_{3} \mathrm{Fe}(\mathrm{CN})_{6}$ flow, and close inlet and outlet valves of the detection chamber before starting the EIS procedure.

? TROUBLESHOOTING 
Table 1 | Exemplary on-chip microelectrode functionalization procedures for EC measurements and differences between those using aptamers and antibodies

\begin{tabular}{|c|c|c|c|c|}
\hline Functionalization step & Cat. no. & $\begin{array}{l}\text { Functionalization conditions } \\
\text { (concentration, incubation time) }\end{array}$ & $\begin{array}{l}\text { Aptamer-based } \\
\text { functionalization }\end{array}$ & $\begin{array}{l}\text { Antibody-based } \\
\text { functionalization }\end{array}$ \\
\hline \multirow[t]{2}{*}{ SAM } & $\begin{array}{l}\text { 11-MUA (Sigma-Aldrich, cat. no. } \\
\text { 450561) }\end{array}$ & $\begin{array}{l}10 \mathrm{mM} \text { dissolved in absolute ethanol, } 1 \mathrm{~h} \\
\text { incubation }\end{array}$ & + & + \\
\hline & $\begin{array}{l}\text { EDC (Sigma-Aldrich, cat. no. E6383), } \\
\text { NHS (Sigma-Aldrich, cat. no.130672) }\end{array}$ & $\begin{array}{l}50 \mathrm{mM} \text { each dissolved in } 50 \mathrm{mM} \text { citric } \\
\text { acid buffer at } \mathrm{pH} 4.5,30 \mathrm{~min} \text { incubation }\end{array}$ & + & + \\
\hline Aptamer & $\begin{array}{l}\text { Amine-functionalized creatine CK-MB } \\
\text { aptamer (OTC Biotech, customized) }\end{array}$ & $10 \mu \mathrm{g} \mathrm{mL} \mathrm{L}^{-1}$ diluted in PBS, $1 \mathrm{~h}$ incubation & + & - \\
\hline SPV & SPV (Sigma-Aldrich, cat. no. 85878) & $10 \mu \mathrm{g} \mathrm{mL} \mathrm{L}^{-1}$ diluted in PBS, $1 \mathrm{~h}$ incubation & - & + \\
\hline Biotinylated Antibody & $\begin{array}{l}\text { Albumin biotinylated antibody and full- } \\
\text { length protein Albumin ELISA kit } \\
\text { (Abcam, ab108788), GST- } \alpha \\
\text { biotinylated antibody and full-length } \\
\text { protein GST- } \alpha \text { ELISA kit (Abcam, } \\
\text { ab173835) }\end{array}$ & $10 \mu \mathrm{g} \mathrm{mL} \mathrm{L}^{-1}$ diluted in PBS, $1 \mathrm{~h}$ incubation & - & + \\
\hline Medium blocking & Corresponding medium & Corresponding medium, $1 \mathrm{~h}$ incubation & + & + \\
\hline
\end{tabular}

156 Open the bioreceptor valve, and flow the bioreceptor solution for $7 \mathrm{~min}$ at $1.0 \mathrm{~mL} \mathrm{~h}$.

157 Incubate bioreceptor for $1 \mathrm{~h}$ at RT.

$\triangle$ CRITICAL STEP There are two possible strategies for functionalization of the microelectrode surface for biorecognition:

- Aptamer-based functionalization: open aptamer bioreceptor solution valve, and flow $10 \mu \mathrm{g} \mathrm{mL}^{-1}$ of amine-functionalized aptamer solution for $7 \mathrm{~min}$ at $1.0 \mathrm{~mL} \mathrm{~h}^{-1}$. We suggest using this strategy for longer shelf life and sensitivity, if desired aptamers are available.

- Antibody-based functionalization: open antibody solution valve, and flow $10 \mu \mathrm{g} \mathrm{mL} \mathrm{m}^{-1}$ of biotinylated antibody solution for $7 \mathrm{~min}$ at $1.0 \mathrm{~mL} \mathrm{~h}^{-1}$.

The antibodies and aptamers presented in Table 1, with their corresponding concentrations and incubation times listed, are examples from prior studies with hepatic and cardiac microtissue-on-achip cultures ${ }^{13,21,22}$. In general, functionalization with antibodies or aptamers of interest (concentrations and times) needs to be optimized in a case-by-case manner.

158 Open the PBS valve, and flow PBS from the main microchannel for $7 \mathrm{~min}$ at $1.0 \mathrm{~mL} \mathrm{~h}^{-1}$.

159 Open the $\mathrm{K}_{3} \mathrm{Fe}(\mathrm{CN})_{6}$ valve, and flow $50 \mathrm{mM} \mathrm{K}{ }_{3} \mathrm{Fe}(\mathrm{CN})_{6}$ solution for $7 \mathrm{~min}$ at $1.0 \mathrm{~mL} \mathrm{~h}^{-1}$.

160 Measure the bioreceptor-modified microelectrode signal via EIS with the parameters listed in Step 142.

$\triangle$ CRITICAL STEP Stop the $\mathrm{K}_{3} \mathrm{Fe}(\mathrm{CN})_{6}$ flow, and close inlet and outlet valves of the detection chamber before starting the EIS procedure.

? TROUBLESHOOTING

161 Open the PBS valve, and flow PBS from the main microchannel for $7 \mathrm{~min}$ at $1.0 \mathrm{~mL} \mathrm{~h}^{-1}$.

162 Open the medium valve, and flow cell culture medium for $7 \mathrm{~min}$ at $1.0 \mathrm{~mL} \mathrm{~h}^{-1}$. Exemplary medium types are provided in the Reagents list and their reconstitution/storage in Reagent Setup. In reality, the medium should match that used for the specific cell culture of desire.

163 Incubate cell culture medium for $1 \mathrm{~h}$ at RT to ensure blocking of the microelectrode surface to eliminate nonspecific binding.

$\triangle$ CRITICAL STEP During blocking step via incubation of cell culture medium, close inlet and outlet valves of detection chamber.

164 Open the PBS valve, and flow PBS from the main microchannel for $7 \mathrm{~min}$ at $1.0 \mathrm{~mL} \mathrm{~h}^{-1}$.

165 Open the $\mathrm{K}_{3} \mathrm{Fe}(\mathrm{CN})_{6}$ valve, and flow $50 \mathrm{mM} \mathrm{K} \mathrm{K}_{3} \mathrm{Fe}(\mathrm{CN})_{6}$ solution for $7 \mathrm{~min}$ at $1.0 \mathrm{~mL} \mathrm{~h}^{-1}$.

166 Measure the microelectrode signal after blocking via EIS with the parameters listed in Step 142.

$\triangle$ CRITICAL STEP Stop the $\mathrm{K}_{3} \mathrm{Fe}(\mathrm{CN})_{6}$ flow, and close inlet and outlet valves of the detection chamber before starting the EIS procedure.

167 Open the PBS valve, and flow PBS from the main microchannel for $7 \mathrm{~min}$ at $1.0 \mathrm{~mL} \mathrm{~h}^{-1}$.

PAUSE POINT Leave the detection chamber filled with PBS. The functionalized EC sensing chip 
is ready for biomarker sampling and quantification (Fig. 1f-i). The functionalized EC sensing chip can be immediately used (if functionalized together with the integrated platform), or can be stored in a parafilm-sealed humidified chamber at $4{ }^{\circ} \mathrm{C}$ for up to 1 month (if functionalized separately from the integrated platform).

\section{On-chip medium sampling and biomarker quantification Timing $\mathbf{1 . 5} \mathbf{h}$}

168 Open bioreactor sampling valves while shutting down the medium return valve to allow sampling for 5-10 $\mathrm{min}$ at the medium flow rate.

169 Incubate the bioreactor medium sample for $1 \mathrm{~h}$ at RT.

$\triangle$ CRITICAL STEP During incubation of the bioreactor medium sample, close inlet and outlet valves of the detection chamber to avoid any potential interference.

170 Open the PBS valve, and flow PBS from the main microchannel for $7 \mathrm{~min}$ at $1.0 \mathrm{~mL} \mathrm{~h}^{-1}$.

171 Open the $\mathrm{K}_{3} \mathrm{Fe}(\mathrm{CN})_{6}$ valve, and flow $50 \mathrm{mM} \mathrm{K} \mathrm{K}_{3} \mathrm{Fe}(\mathrm{CN})_{6}$ solution for $7 \mathrm{~min}$ at $1.0 \mathrm{~mL} \mathrm{~h}^{-1}$.

172 Measure the captured biomarker signal via EIS with the parameters as listed in Step 142.

$\triangle$ CRITICAL STEP Stop the $\mathrm{K}_{3} \mathrm{Fe}(\mathrm{CN})_{6}$ flow, and close inlet and outlet valves of the detection chamber before starting the EIS procedure.

\section{? TROUBLESHOOTING}

173 Open the PBS valve, and flow PBS from the main microchannel for $7 \mathrm{~min}$ at $1.0 \mathrm{~mL} \mathrm{~h}^{-1}$.

174 Repeat Steps 167-171 for repeated biomarker measurements until the biosensor surface saturates. ? TROUBLESHOOTING

175 Regenerate the biosensor surface by a two-step cleaning procedure. Follow Steps 175-177 listed below.

\section{On-chip EC affinity-based biosensor regeneration Timing 40 min}

176 Perform the first step of EC cleaning by opening the $\mathrm{H}_{2} \mathrm{SO}_{4}$ valve and flowing the $10 \mathrm{mM} \mathrm{H}_{2} \mathrm{SO}_{4}$ solution for $7 \mathrm{~min}$ at $1.0 \mathrm{~mL} \mathrm{~h}^{-1}$, followed by an additional $7 \mathrm{~min}$ flow under the $\mathrm{CV}$ potential sweep with the CV parameters of:

\begin{tabular}{ll} 
Parameter & Value \\
\hline Initial E (V) & 0.0 \\
High E (V) & 1.8 \\
Low E (V) & 0.0 \\
Final E (V) & 0.0 \\
Initial scan polarity & Positive \\
Scan rate $\left(\mathrm{V} \mathrm{s}^{-1}\right)$ & 0.1 \\
Sweep segments & 8 \\
Sample interval (V) & 0.001 \\
Quiet time $(\mathrm{sec})$ & 2 \\
Sensitivity $\left(\mathrm{A} \mathrm{V} \mathrm{V}^{-1}\right)$ & $1.0 \times 10^{-0.002}$ \\
Communication port speed & Fast \\
Line frequency & $60 \mathrm{~Hz}$ \\
Potential axis & Positive left
\end{tabular}

$\triangle$ CRITICAL STEP Do not stop the $\mathrm{H}_{2} \mathrm{SO}_{4}$ flow during EC cleaning.

177 Open the PBS valve, and flow PBS from the main microchannel for $7 \mathrm{~min}$ at $1.0 \mathrm{~mL} \mathrm{~h}^{-1}$.

178 Perform the second step of EC cleaning by opening the $\mathrm{K}_{3} \mathrm{Fe}(\mathrm{CN})_{6}$ valve and flowing the $50 \mathrm{mM}$ $\mathrm{K}_{3} \mathrm{Fe}(\mathrm{CN})_{6}$ solution for $7 \mathrm{~min}$ at $1.0 \mathrm{~mL} \mathrm{~h}^{-1}$, followed by additional $7 \mathrm{~min}$ flow under the $\mathrm{CV}$ potential sweep with the $\mathrm{CV}$ parameters of:

\begin{tabular}{lc} 
Parameter & Value \\
\hline Initial E (V) & 1.0 \\
High E (V) & 1.2 \\
Low E (V) & -1.2 \\
Final E (V) & 0.0 \\
& Table continued
\end{tabular}




\begin{tabular}{ll} 
(continued) & \\
Parameter & Value \\
\hline Initial scan polarity & Negative \\
Scan rate $\left(\mathrm{V} \mathrm{s}^{-1}\right)$ & 0.2 \\
Sweep segments & 6 \\
Sample interval $(\mathrm{V})$ & 0.002 \\
Quiet time $(\mathrm{sec})$ & 0 \\
Sensitivity $\left(\mathrm{A} \mathrm{V}^{-1}\right)$ & $1.0 \times 10^{-0.004}$ \\
Communication port speed & Fast \\
Line frequency & $60 \mathrm{~Hz}$ \\
Potential axis & Positive left \\
&
\end{tabular}

$\triangle$ CRITICAL STEP Do not stop the $\mathrm{K}_{3} \mathrm{Fe}(\mathrm{CN})_{6}$ flow during EC cleaning.

179 Measure the bare microelectrode signal via CV with the parameters as follows:

\begin{tabular}{ll} 
Parameter & Value \\
\hline Initial E (V) & 0.0 \\
High E (V) & 0.6 \\
Low E (V) & -0.6 \\
Final E (V) & 0.0 \\
Initial scan polarity & Negative \\
Scan rate $\left(\mathrm{V} \mathrm{s}^{-1}\right)$ & 0.075 \\
Sweep segments & 6 \\
Sample interval (V) & 0.001 \\
Quiet time $(\mathrm{sec})$ & 0 \\
Sensitivity $\left(\mathrm{A} \mathrm{V} \mathrm{V}^{-1}\right)$ & $1.0 \times 10^{-0.004}$ \\
Communication port speed & Fast \\
Line frequency & $60 \mathrm{~Hz}$ \\
Potential axis & Positive left
\end{tabular}

$\triangle$ CRITICAL STEP Stop the $\mathrm{K}_{3} \mathrm{Fe}(\mathrm{CN})_{6}$ flow, and close inlet and outlet valves of the detection chamber before starting the $\mathrm{CV}$ procedure.

180 Measure the bare microelectrode signal via EIS with the parameters as follows:

\begin{tabular}{ll} 
Parameter & Value \\
\hline Initial E $(\mathrm{V})$ & 0.0 \\
High frequency $(\mathrm{Hz})$ & 100,000 \\
Low frequency $(\mathrm{Hz})$ & 0.1 \\
Amplitude $(\mathrm{V})$ & 0.05 \\
Quiet time $(\mathrm{sec})$ & 0 \\
Sensitivity $\left(\mathrm{A} \mathrm{V}^{-1}\right)$ & $1.0 \times 10^{-0.004}$ \\
Bias DC current during run & Below $1 \mathrm{~Hz}$
\end{tabular}

181 Once regenerated, refunctionalization of the microelectrode for biomarker detections can be performed by iterating Steps 143-174.

$\triangle$ CRITICAL STEP Regenerations and detections will be limited by the thickness of the microelectrodes (Fig. 2) and the total volumes of detection solutions available. For continued biosensing when EC sensing chips can no longer be used, they can be modularly replaced, while the breadboard and microbioreactors can carry on working.

\section{? TROUBLESHOOTING}




\section{Troubleshooting}

Troubleshooting advice can be found in Table 2 .

\section{Table 2 | Troubleshooting table}

\begin{tabular}{lll} 
Step & Problem & Possible reason \\
\hline 57 & $\begin{array}{l}\text { Failure to achieve } \\
\text { negative membrane } \\
\text { deformation }\end{array}$ & Vacuum tubing blocked \\
& & $\begin{array}{l}\text { Tubing has been inserted too deep into the } \\
\text { inlet/outlet and is proximal to the membrane } \\
\text { Vacuum tubing blocked by SU-8 or PDMS } \\
\text { residues } \\
\text { PDMS membrane laceration }\end{array}$ \\
& $\begin{array}{l}\text { Faulty valve channel } \\
\text { filling }\end{array}$ & Presence of gas in the valve channel
\end{tabular}

No movement of the liquid in the valve channel

Tubing leakage

Tubing blockage

SPV was degraded (due to long-term storage of SPV at $-20^{\circ} \mathrm{C}$ )

SAM formation was not successful. EDC/NHS coupling (long-term storage of EDC/NHS) did not work

SAM formation was not successful. 11-MUA covalent bonding did not work (improper storage, contamination by other liquids and resulting evaporation might change the 11-MUA concentration)

Bioreceptor was degraded (long-term storage) Incubation temperature was not appropriate

Bioreceptor was degraded (long-term storage)

Sampling frequency was very high, and biomarker secretion level was out of biosensor sensitivity range

Electrode surface was damaged

Regeneration was not successful. $\mathrm{K}_{3} \mathrm{Fe}(\mathrm{CN})_{6}$ or $\mathrm{H}_{2} \mathrm{SO}_{4}$ flow was stopped during $\mathrm{EC}$ regeneration

Regeneration was not successful. $\mathrm{K}_{3} \mathrm{Fe}(\mathrm{CN})_{6}$ solution was deteriorated (long-term and/or wrong storage without light protection)

\section{Solution}

Visually evaluate all tubing and connections from pump inlet to PDMS chip. Exchange any leaky or block section

Pull tubing partially, with pump turned off

Exchange blocked section with new tubing

This is a terminal situation. Create a new square PDMS mold with a membrane. Repeat Steps 43-56

Continue to purge gas into the valve channel until it diffuses through the PDMS slabs

Partially increase solenoid by 1 PSI at a time slowly. Do not exceed the 15 PSI pressure or the device might explode

Evaluate if the membrane is bound into channel or PDMS debris is inside it. Terminate the chip, and repeat Steps 65-95

Change the O-ring of the reservoir lid

Add more 5 min epoxy resin mix on top of the reservoir lid

Change the base of the reservoir

If issue continues, repeat Steps 96-103

Bypass tubing laceration with metal connectors

If issue continues, repeat Steps 96-103

Increase solenoid pressure by 2-5 PSI to remove blockage

Bypass tubing blockage with metal connectors

If issue continues, repeat Steps 96-103

Prepare a new batch of SPV solution, and follow Steps 154-159

Prepare a new batch of EDC/NHS solution. Incubate SPV, and measure the signal afterwards by following Steps 148-154

If the $R_{\mathrm{ct}}$ value of SMA layer does not substantially increase, prepare new 11-MUA solution and restart SAM formation by following Steps 124-145

Prepare a new batch of stock bioreceptor solution Check the ambient air temperature, and adjust the temperature accordingly

Prepare a new batch of stock bioreceptor solution Increase the sampling interval

The number of regenerations exceeded maximum; start with a new microelectrode

Check the valves and tubing and ensure free flow

Prepare a new $\mathrm{K}_{3} \mathrm{Fe}(\mathrm{CN})_{6}$ stock solution 


\section{NATURE PROTOCOLS}

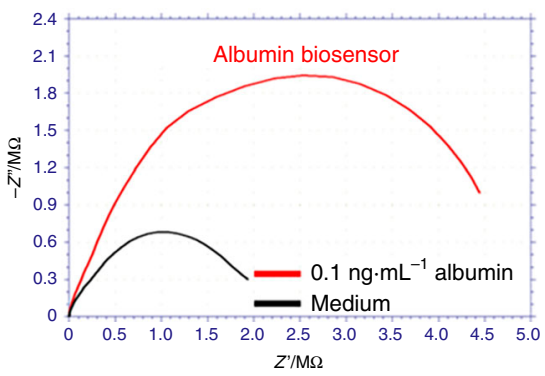

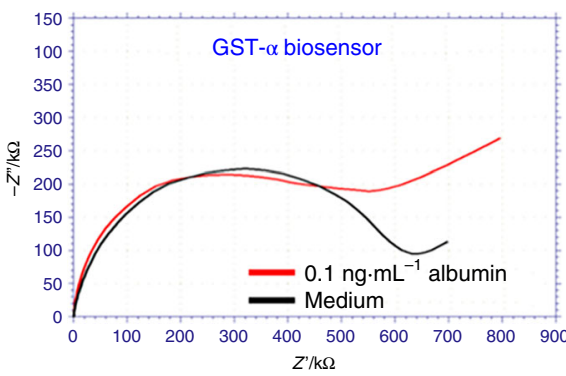

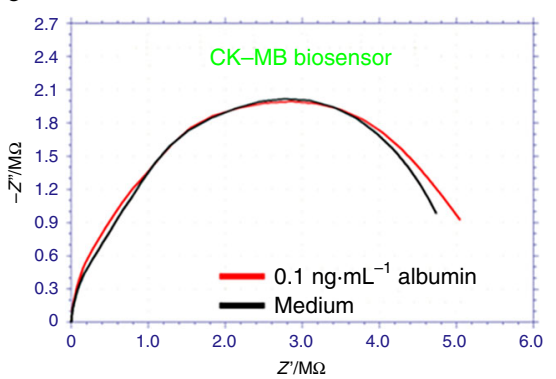

d Human heart-liver-on-chips (APAP)

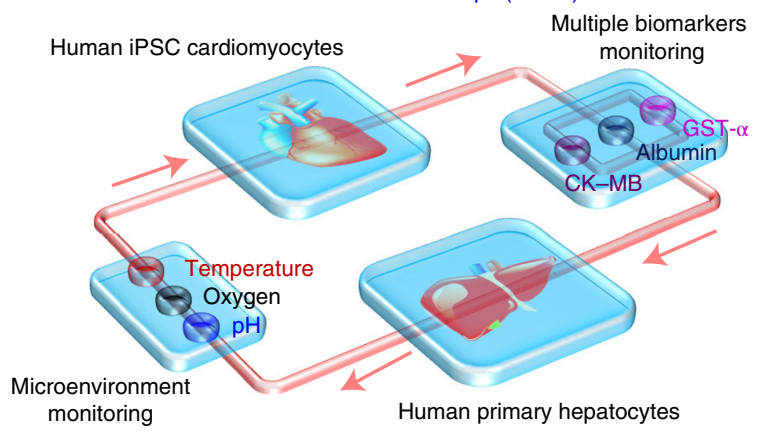

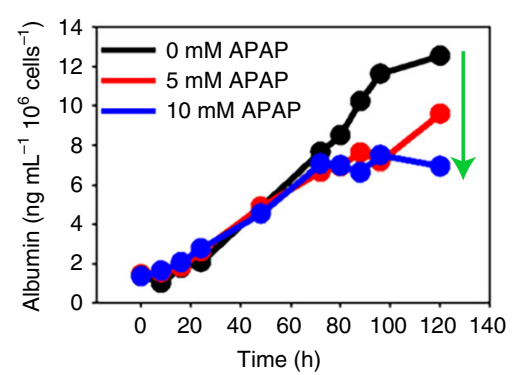

f

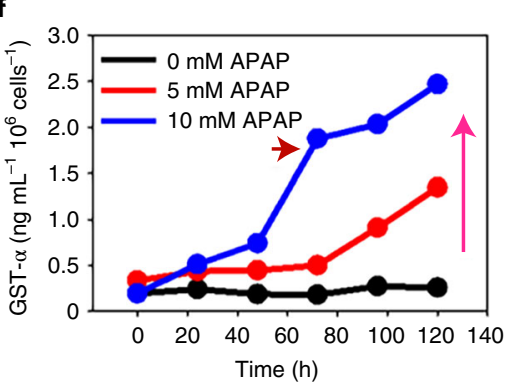

g

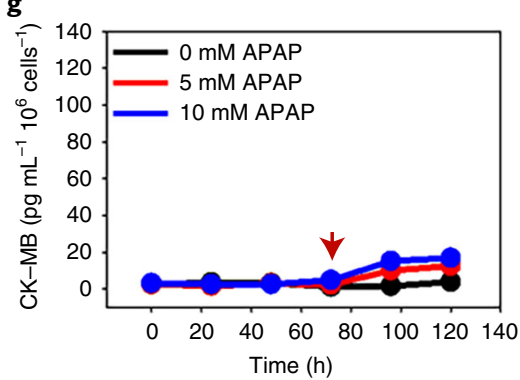

h

Human heart-liver-cancer-on-chips (DOX)

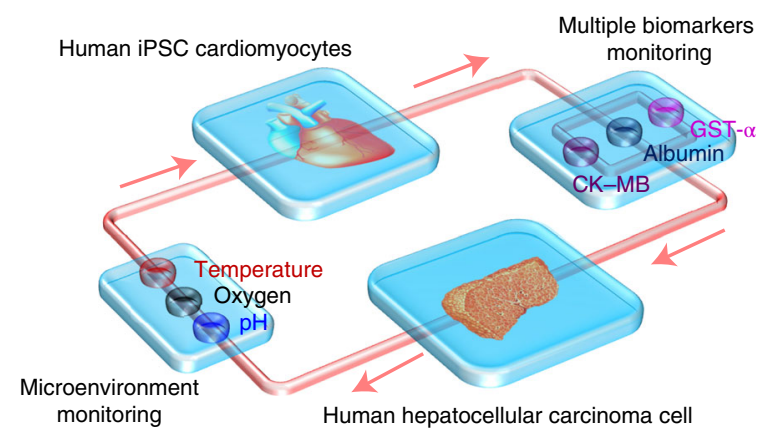

i

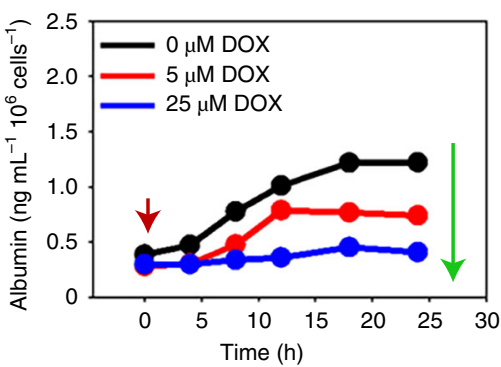

j

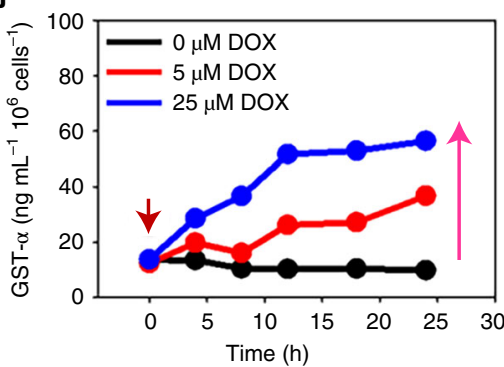

k

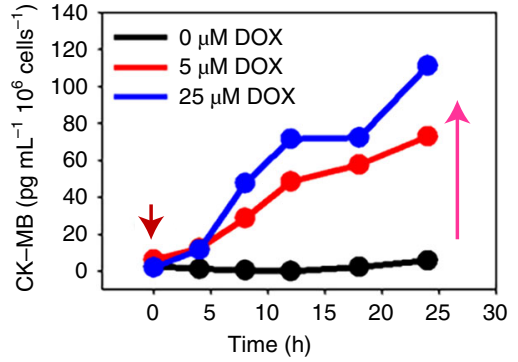


Fig. 5 | Examples of EC affinity-based biosensor detection capabilities in multi-organ-on-a-chip configurations. a-c, Impedance signals from electrodes primed with albumin (a), GST- $\alpha$ (b) and CK-MB bioreceptors (c), exposed to control medium and medium spiked with albumin. d, Breadboard-connected dual-organ system with a human primary hepatic bioreactor and a human induced pluripotent stem cell-derived cardiac bioreactor exposed to acetaminophen (APAP), where dynamic sampling of the medium into the EC affinity-based biosensor chips yields in-line multiplexed biomarker detections for up to $120 \mathrm{~h}$. Changes in concentrations of albumin (green arrow) and GST- $\alpha$ (pink arrow) as well as drug administration points (small red arrows) are indicated. e,f, APAP hepatotoxicity was demonstrated by reduced albumin secretion and increased GST- $\alpha$ secretion following drug infusion. $\mathbf{g}$, Cardiotoxicity was measured by the release of CK-MB from impaired cardiomyocytes, where chronic cardiac damage from APAP toxicity was barely detected. h, Breadboard-connected dual-organ system with a human hepatic cancer bioreactor and a human induced pluripotent stem cell-derived cardiac bioreactor exposed to doxorubicin (DOX), where dynamic sampling of the medium into the EC affinitybased biosensor chips yields in-line multiplexed biomarker detections for up to $24 \mathrm{~h}$. i,j, DOX toxicity on hepatic cancer cells was demonstrated by reduced albumin secretion (green arrow) and increased GST- $\alpha$ secretion (pink arrow) following drug infusion. Drug administration points are indicated by small red arrows. k, Side cardiotoxicity was measured by the release of CK-MB (pink arrow) from injured cardiomyocytes, where acute cardiac damage was noticeable, $n=1$. Figure adapted with permission from ref. ${ }^{13}$.

\section{Timing}

Steps 1-10, Microelectrode fabrication: $5 \mathrm{~h}$

Steps 11-31, Photolithographic fabrication of microfluidic controlling devices: $18 \mathrm{~h}$

Steps 31-35, PDMS membrane fabrication: $1.5 \mathrm{~h}$

Steps 36-42, Fluidic layer fabrication: $3 \mathrm{~h}$

Steps 43-64, Hemicylindrical channels fabrication: $16 \mathrm{~h}$

Steps 65-86, EC detection chip assembly: $20 \mathrm{~h}$

Steps 87-95, EC detection chip and sensor incorporation: $1 \mathrm{~h}$

Steps 96-103, Reservoir fabrication: $1.5 \mathrm{~h}$

Steps 104-108, Breadboard assembly: $20 \mathrm{~h}$

Steps 109-120, Automation operational system preparation: $1.5 \mathrm{~h}$

Steps 121-136, Microbioreactors and breadboard fluidics integration: $1.5 \mathrm{~h}$

Steps 137-166, Functionalization of the microfluidic EC sensing chip: $6 \mathrm{~h}$

Steps 167-174, On-chip medium sampling and biomarker quantification: $1.5 \mathrm{~h}$

Steps 175-180, On-chip affinity-based biosensor regeneration: $40 \mathrm{~min}$

\section{Anticipated results}

Integration of aptamer-based and antibody-based microfluidic EC affinity-based biosensors into organ-on-a-chip platforms enables quantification of protein biomarker secretion and, hence, monitoring not only of the effect of drug toxicity but also of how cell fate and viability are affected by various parameters in the cell culture microenvironment. Thanks to specific functionalization of the microelectrode surface and the two-step regeneration strategy, nanomolar-range multiplexed detection of biomarkers can be achieved for lengthy periods with high selectivity (Fig. 5) ${ }^{13,15}$. The label-free detection strategy based on charge transfer resistance measurement is versatile and could be applied to all biomarkers, providing that commercial bioreceptor molecules exist, or that aptamers can be synthesized via the SELEX process. After successful functionalization of the microelectrode, $R_{\mathrm{ct}}$ values increase by $\sim 2$ orders of magnitude and continue to increase after each successive step (Fig. 1c,d,f,h) until the electrode surface is saturated, where the two-step regeneration is applied to revert back to the bare microelectrode signal (Fig. 1e, Fig. 2). The cell culture medium blocking step is an essential step for the calibration of the affinity-based biosensors and, depending on the medium composition, that is, the amount and type of soluble proteins and other biological agents, different $R_{\mathrm{ct}}$ values may be obtained. Hence, normalization of the measured $R_{\mathrm{ct}}$ values for target biomarkers with respect to the $R_{\mathrm{ct}}$ recorded in the medium blocking step is necessary.

In a few practical examples, we have previously shown that our EC affinity-based biosensors could be conveniently integrated with dual-organ-on-chips platforms while showing high selectivity and no crosstalk for biomarker detection (Fig. 5a-c). Pharmaceutical compound-induced chronic hepatotoxicity and minimum impact on cardiac functions were readily picked up in a human heart-liver-onchips configuration (Fig. $5 \mathrm{~d}-\mathrm{g}$ ). Similarly, anticancer drug treatment efficacy on and simultaneous side cardiotoxicity were successfully monitored in a human heart-liver cancer-on-chips configuration (Fig. 5h-j). It is worth noting that, due to the noninvasiveness of our EC affinity-based biosensing mechanism, the data shown in Fig. $5 \mathrm{e}-\mathrm{g}$, $\mathrm{i}-\mathrm{k}$ were based on their respective individual dual-organ platforms, continually monitored by dynamically sampling the media at predefined time points. This ability to monitor an individual organ pair over extended periods is critical, especially for future 
personalized medicine, since with conventional endpoint assays average values will have to be taken from multiple samples that might either bias the results or require larger sample numbers that are not always feasible. Nevertheless, results derived from parallel systems might still be averaged to understand the collective statistical effects of the pharmaceutical compounds applied to the organ models, as also shown in our previous work ${ }^{13}$.

Antibody- and aptamer-based functionalization strategies have their own advantages and disadvantages. Aptamers provide better sensitivity than antibodies in complex biological media containing large amounts of nonspecific biomolecules since they do not bind to serum proteins and have higher binding affinities to their target molecules. Moreover, aptamers enable longer shelf lives than antibodies because of their stability at varying temperatures, while antibodies denature at elevated temperatures. However, the development of specific aptamers for a wide range of targets still represents a challenge, whereas antibody production is a routine protocol and thus has much better availability. In addition, aptamers are prone to degradation of nucleases if not modified, which might hamper the use of aptamers for detection in blood or media containing nucleases ${ }^{39,40}$.

\section{Reporting Summary}

Further information on research design is available in the Nature Research Reporting Summary linked to this article.

\section{Data availability}

Most data associated with this protocol can be found in previous publications ${ }^{13,21,22}$. Additional datasets that support this protocol are available from the corresponding authors upon reasonable request. All requests for raw and analyzed data and materials will be promptly reviewed by the Brigham and Women's Hospital to verify whether the request is subject to any intellectual property or confidentiality obligations. Any data and materials that can be shared will be released via a Material Transfer Agreement.

\section{Code availability}

The MATLAB codes can be accessed through previous publications ${ }^{13,21,22}$ and the Zhang Lab website (Electrochemical Biosensing; https://shrikezhang.com/publications/opensource). The running of these codes is standardized, and additional instructions can be found in the readme file in the package.

\section{References}

1. Ahadian, S. et al. Organ-on-a-chip platforms: a convergence of advanced materials, cells, and microscale technologies. Adv. Healthc. Mater. https://doi.org/10.1002/adhm.201800734 (2018).

2. Wang, Y. I., Carmona, C., Hickman, J. J. \& Shuler, M. L. Multiorgan microphysiological systems for drug development: strategies, advances, and challenges. Adv. Healthc. Mater. 7, 1701000 (2018).

3. Ronaldson-Bouchard, K. \& Vunjak-Novakovic, G. Organs-on-a-chip: a fast track for engineered human tissues in drug development. Cell Stem Cell 22, 310-324 (2018).

4. Zhang, Y. S. et al. From cardiac tissue engineering to heart-on-a-chip: beating challenges. Biomed. Mater. 10, 034006 (2015).

5. Arrigoni, C., Lopa, S., Candrian, C. \& Moretti, M. Organs-on-a-chip as model systems for multifactorial musculoskeletal diseases. Curr. Opin. Biotechnol. 63, 79-88 (2020).

6. Mobini, S., Song, Y. H., McCrary, M. W. \& Schmidt, C. E. Advances in ex vivo models and lab-on-a-chip devices for neural tissue engineering. Biomaterials 198, 146-166 (2019).

7. Bein, A. et al. Microfluidic organ-on-a-chip models of human intestine. Cell. Mol. Gastroenterol. Hepatol. 5, 659-668 (2018).

8. Ashammakhi, N., Wesseling-Perry, K., Hasan, A., Elkhammas, E. \& Zhang, Y. S. Kidney-on-a-chip: untapped opportunities. Kidney Int. 94, 1073-1086 (2018).

9. Conant, G. et al. High-content assessment of cardiac function using heart-on-a-chip devices as drug screening model. Stem Cell Rev. Rep. 13, 335-346 (2017).

10. Ribas, J. et al. Biomechanical strain exacerbates inflammation on a progeria-on-a-chip model. Small https:// doi.org/10.1002/smll.201603737 (2017).

11. Zhang, Y. S., Zhang, Y. N. \& Zhang, W. Cancer-on-a-chip systems at the frontier of nanomedicine. Drug Discov. Today 22, 1392-1399 (2017).

12. Skardal, A., Shupe, T. \& Atala, A. Organoid-on-a-chip and body-on-a-chip systems for drug screening and disease modeling. Drug Discov. Today 21, 1399-1411 (2016).

13. Zhang, Y. S. et al. Multisensor-integrated organs-on-chips platform for automated and continual in situ monitoring of organoid behaviors. Proc. Natl Acad. Sci. USA 114, E2293-E2302 (2017). 
14. Riahi, R. et al. Automated microfluidic platform of bead-based electrochemical immunosensor integrated with bioreactor for continual monitoring of cell secreted biomarkers. Sci. Rep. 6, 24598 (2016).

15. Skardal, A. et al. Multi-tissue interactions in an integrated three-tissue organ-on-a-chip platform. Sci. Rep. 7 , 8837 (2017).

16. Skardal, A. et al. Drug compound screening in single and integrated multi-organoid body-on-a-chip systems. Biofabrication 12, 025017 (2020).

17. Edington, C. D. et al. Interconnected microphysiological systems for quantitative biology and pharmacology studies. Sci. Rep. 8, 4530 (2018).

18. Oleaga, C. et al. Multi-organ toxicity demonstration in a functional human in vitro system composed of four organs. Sci. Rep. 6, 20030 (2016).

19. Novak, R. et al. Robotic fluidic coupling and interrogation of multiple vascularized organ chips. Nat. Biomed. Eng. 4, 407-420 (2020).

20. Kratz, S. R. A., Holl, G., Schuller, P., Ertl, P. \& Rothbauer, M. Latest trends in biosensing for microphysiological organs-on-a-chip and body-on-a-chip systems. Biosensors (Basel) https://doi.org/10.3390/ bios9030110 (2019).

21. Shin, S. R. et al. Label-free and regenerative electrochemical microfluidic biosensors for continual monitoring of cell secretomes. Adv. Sci. (Weinh.) 4, 1600522 (2017).

22. Shin, S. R. et al. Aptamer-based microfluidic electrochemical biosensor for monitoring cell-secreted trace cardiac biomarkers. Anal. Chem. 88, 10019-10027 (2016).

23. Zhang, Y. S. et al. Google Glass-directed monitoring and control of microfluidic biosensors and actuators. Sci. Rep. 6, 22237 (2016)

24. Rusling, J. F. Multiplexed electrochemical protein detection and translation to personalized cancer diagnostics. Anal. Chem. 85, 5304-5310 (2013).

25. Goode, J. A., Rushworth, J. V. H. \& Millner, P. A. Biosensor regeneration: a review of common techniques and outcomes. Langmuir 31, 6267-6276 (2015).

26. Rajan, S. A. P. et al. Probing prodrug metabolism and reciprocal toxicity with an integrated and humanized multi-tissue organ-on-a-chip platform. Acta Biomater. 106, 124-135 (2020).

27. Aleman, J. \& Skardal, A. A multi-site metastasis-on-a-chip microphysiological system for assessing metastatic preference of cancer cells. Biotechnol. Bioeng. 116, 936-944 (2019).

28. Aleman, J. et al. Deconstructed microfluidic bone marrow on-a-chip to study normal and malignant hemopoietic cell-niche interactions. Small 15, e1902971 (2019).

29. Soucy, J. R., Bindas, A. J., Koppes, A. N. \& Koppes, R. A. Instrumented microphysiological systems for realtime measurement and manipulation of cellular electrochemical processes. IScience 21, 521-548 (2019).

30. Pourmand, A. et al. Fabrication of whole-thermoplastic normally closed microvalve, micro check valve, and micropump. Sens. Actuat. B-Chem. 262, 625-636 (2018).

31. Shaegh, S. A. M. et al. Rapid prototyping of whole-thermoplastic microfluidics with built-in microvalves using laser ablation and thermal fusion bonding. Sens. Actuat. B-Chem. 255, 100-109 (2018).

32. Lee, Y.-S., Bhattacharjee, N. \& Folch, A. 3D-printed Quake-style microvalves and micropumps. Lab Chip 18, 1207-1214 (2018).

33. Ellington, A. D. \& Szostak, J. W. In vitro selection of RNA molecules that bind specific ligands. Nature 346 , 818-822 (1990).

34. Dauphin-Ducharme, P., Arroyo-Currás, N. Y. \& Plaxco, K. W. High-precision electrochemical measurements of the guanine-, mismatch-, and length-dependence of electron transfer from electrode-bound DNA are consistent with a contact-mediated mechanism. J. Am. Chem. Soc. 141, 1304-1311 (2019).

35. Kurnik, M., Pang, E. Z. \& Plaxco, K. W. An electrochemical biosensor architecture based on protein folding supports direct real-time measurements in whole blood. Angew. Chem. (2020).

36. Kang, E., Shin, S.-J., Lee, K. H. \& Lee, S.-H. Novel PDMS cylindrical channels that generate coaxial flow, and application to fabrication of microfibers and particles. Lab Chip 10, 1856-1861 (2010).

37. Kang, E. et al. Digitally tunable physicochemical coding of material composition and topography in continuous microfibres. Nat. Mater. 10, 877-883 (2011).

38. MacDonald, M. A. \& Andreas, H. A. Method for equivalent circuit determination for electrochemical impedance spectroscopy data of protein adsorption on solid surfaces. Electrochim. Acta 129, 290-299 (2014).

39. McCauley, T. G., Hamaguchi, N. \& Stanton, M. Aptamer-based biosensor arrays for detection and quantification of biological macromolecules. Anal. Biochem. 319, 244-250 (2003).

40. Kara, P. et al. Aptamers based electrochemical biosensor for protein detection using carbon nanotubes platforms. Biosens. Bioelectron. 26, 1715-1718 (2010).

\section{Acknowledgements}

The authors acknowledge support by the National Institutes of Health (R00CA201603 and UG3TR003274) and the Brigham Research Institute. This work was also partially sponsored by the Office of the Secretary of Defense through the Advanced Regenerative Manufacturing Institute (ARMI|BioFabUSA) and was accomplished under Agreement Number W911NF-17-3-003. The views and conclusions contained in this document are those of the authors and should not be interpreted as representing the official policies, either expressed or implied, of the Office of the Secretary of Defense or the US Government. The US Government is authorized to reproduce and distribute reprints for government purposes notwithstanding any copyright notation here. T.K. is grateful to the Swiss National Science Foundation for the Postdoc Mobility fellowship (no. P400PM_180788/1). 


\section{Author contributions}

Y.S.Z. and S.R.S. conceived the project; J.A., T.K. and Y.S.Z. drafted the initial manuscript; L.S.M. created the figures; J.A., T.K., S.R.S. and Y.S.Z. revised the manuscript. All authors approved the manuscript.

\section{Competing interests}

The authors declare no competing interests.

\section{Additional information}

Supplementary information The online version contains supplementary material available at https://doi.org/10.1038/s41596-021-00511-7. Correspondence and requests for materials should be addressed to S.R.S. or Y.S.Z.

Peer review information Nature Protocols thanks Xingyu Jiang, Arben Merkoçi and José Pingarrón for their contribution to the peer review of this work.

Reprints and permissions information is available at www.nature.com/reprints.

Publisher's note Springer Nature remains neutral with regard to jurisdictional claims in published maps and institutional affiliations.

Received: 3 September 2020; Accepted: 22 January 2021;

Published online: 28 April 2021

\section{Related links}

Key reference using this protocol:

Zhang, Y. S. et al. Proc. Natl Acad. Sci. USA 114, E2293-E2302 (2017): https://doi.org/10.1073/pnas.1612906114

Shin, S. R. et al. Adv. Sci. (Weinh) 4, 1600522 (2017): https://doi.org/10.1002/advs.201600522

Shin, S. R. et al. Anal. Chem. 88, 10019-10027 (2016): https://doi.org/10.1021/acs.analchem.6b02028 


\section{Reporting Summary}

Nature Research wishes to improve the reproducibility of the work that we publish. This form provides structure for consistency and transparency in reporting. For further information on Nature Research policies, see our Editorial Policies and the Editorial Policy Checklist.

\section{Statistics}

For all statistical analyses, confirm that the following items are present in the figure legend, table legend, main text, or Methods section.

n/a Confirmed

$\square$ \The exact sample size $(n)$ for each experimental group/condition, given as a discrete number and unit of measurement

Х $\square$ A statement on whether measurements were taken from distinct samples or whether the same sample was measured repeatedly

$\triangle$ The statistical test(s) used AND whether they are one- or two-sided

Х $\square$ Only common tests should be described solely by name; describe more complex techniques in the Methods section.

Х $\square$ A description of all covariates tested

Х $\square$ A description of any assumptions or corrections, such as tests of normality and adjustment for multiple comparisons

$\square$ A full description of the statistical parameters including central tendency (e.g. means) or other basic estimates (e.g. regression coefficient)

$\triangle \square$ AND variation (e.g. standard deviation) or associated estimates of uncertainty (e.g. confidence intervals)

$\bigotimes \square \begin{aligned} & \text { For null hypothesis testing, the test statistic (e.g. } F, t, r \text { ) with confidence intervals, effect sizes, degrees of freedom and } P \text { value noted } \\ & \text { Give } P \text { values as exact values whenever suitable. }\end{aligned}$

Х $\square$ For Bayesian analysis, information on the choice of priors and Markov chain Monte Carlo settings

Х $\square$ For hierarchical and complex designs, identification of the appropriate level for tests and full reporting of outcomes

\ $\square$ Estimates of effect sizes (e.g. Cohen's $d$, Pearson's $r$ ), indicating how they were calculated

Our web collection on statistics for biologists contains articles on many of the points above.

\section{Software and code}

Policy information about availability of computer code

Data collection The MATLAB codes can be accessed through key references and the Zhang Lab website (https://shrikezhang.com/publications/opensource).

Data analysis No new data were collected and they were referenced from the key references. Full descriptions were presented in the key references.

For manuscripts utilizing custom algorithms or software that are central to the research but not yet described in published literature, software must be made available to editors and reviewers. We strongly encourage code deposition in a community repository (e.g. GitHub). See the Nature Research guidelines for submitting code \& software for further information.

\section{Data}

Policy information about availability of data

All manuscripts must include a data availability statement. This statement should provide the following information, where applicable:

- Accession codes, unique identifiers, or web links for publicly available datasets

- A list of figures that have associated raw data

- A description of any restrictions on data availability

Most data associated with this protocol can be found in the Key References. Additional datasets that support this protocol are available from the corresponding authors upon reasonable request. All requests for raw and analyzed data and materials will be promptly reviewed by the Brigham and Women's Hospital to verify whether the request is subject to any intellectual property or confidentiality obligations. Any data and materials that can be shared will be released via a Material Transfer Agreement. 


\section{Field-specific reporting}

Please select the one below that is the best fit for your research. If you are not sure, read the appropriate sections before making your selection. $\bigotimes$ Life sciences $\quad \square$ Behavioural \& social sciences $\quad \square$ Ecological, evolutionary \& environmental sciences

For a reference copy of the document with all sections, see nature.com/documents/nr-reporting-summary-flat.pdf

\section{Life sciences study design}

All studies must disclose on these points even when the disclosure is negative.

Sample size Performance of independent experiments were set up in at least three or more replicates, based on a sample size calculated using power analysis from anticipated results (means and standard deviations) from the key references.

Zhang, Y.S., et al. Multisensor-integrated organs-on-chips platform for automated and continual in situ monitoring of organoid behaviors. Proc Natl Acad Sci U S A 114, E2293-E2302 (2017). DOI: 10.1073/pnas.1612906114.

Shin, S.R., et al. Label-Free and Regenerative Electrochemical Microfluidic Biosensors for Continual Monitoring of Cell Secretomes. Adv Sci (Weinh) 4, 1600522 (2017). DOI:10.1002/advs.201600522.

Shin, S.R., et al. Aptamer-Based Microfluidic Electrochemical Biosensor for Monitoring Cell-Secreted Trace Cardiac Biomarkers. Anal Chem 88, 10019-10027 (2016). DOI:10.1021/acs.analchem.6b02028.

Data exclusions For electrochemical measurements the samples were excluded from the analysis when leakages from the sensing chips were observed; otherwise, all the data were included.

Replication Fully functional sensors, electrochemical chips, and breadboard have to be thoroughly evaluated before running the experiment to ensure replication. The experimental reagents should only be used during the established time frame after preparation and if stored under the appropriate conditions.

Randomization In this article, we present the protocol for the development of a electrochemically affinity based biosensor. Validation of biomarker sensitivity and specificity has been previously reported.

Zhang, Y.S., et al. Multisensor-integrated organs-on-chips platform for automated and continual in situ monitoring of organoid behaviors. Proc Natl Acad Sci U S A 114, E2293-E2302 (2017). DOI: 10.1073/pnas.1612906114.

Shin, S.R., et al. Label-Free and Regenerative Electrochemical Microfluidic Biosensors for Continual Monitoring of Cell Secretomes. Adv Sci (Weinh) 4, 1600522 (2017). DOI:10.1002/advs.201600522.

Shin, S.R., et al. Aptamer-Based Microfluidic Electrochemical Biosensor for Monitoring Cell-Secreted Trace Cardiac Biomarkers. Anal Chem 88, 10019-10027 (2016). DOI:10.1021/acs.analchem.6b02028.

\section{Reporting for specific materials, systems and methods}

We require information from authors about some types of materials, experimental systems and methods used in many studies. Here, indicate whether each material, system or method listed is relevant to your study. If you are not sure if a list item applies to your research, read the appropriate section before selecting a response.

Materials \& experimental systems

\section{n/a}

n/a $\mid$ Involved in the study

Methods

$\square$ Antibodies

Х $\square$ Eukaryotic cell lines

Х $\square$ Palaeontology and archaeology

$\bigotimes \square$ Animals and other organisms

$\bigotimes \square$ Human research participants

$\bigotimes \square$ Clinical data

$\bigotimes \square$ Dual use research of concern

\section{Antibodies}

Antibodies used Albumin biotinylated antibody and full-length protein from Albumin ELISA kit (Abcam cat. no. ab108788, RRID: AB_2876883); Glutathione S-transferase-alpha (GST- $\alpha$ ) biotinylated antibody and full-length protein from GST- $\alpha$ ELISA kit (Abcam, cat. no. ab173835, RRID: AB_2876882); CK-MB full-length protein from CK-MB ELISA kit (Abcam, ab193696, RRID: AB_2876884) 
Validation

validated by our team in the paper: Shin, S.R., et al. Label-Free and Regenerative Electrochemical Microfluidic Biosensors for Continual Monitoring of Cell Secretomes. Adv Sci (Weinh) 4, 1600522 (2017).

We reported the multiplexed electrochemical biosensor capability of continuous detection of the same antibodies in the paper: Chang, Y.S., et al. Multisensor-integrated organs-on-chips platform for automated and continual in situ monitoring of organoid behaviors. Proc Natl Acad Sci U S A 114, E2293-E2302 (2017). 